\title{
Emergent gravity on covariant quantum spaces in the IKKT model
}

\author{
Harold C. Steinacker \\ Faculty of Physics, University of Vienna, \\ Boltzmanngasse 5, A-1090 Vienna, Austria \\ E-mail: harold.steinacker@univie.ac.at
}

ABSTRACT: We study perturbations of 4-dimensional fuzzy spheres as backgrounds in the IKKT or IIB matrix model. Gauge fields and metric fluctuations are identified among the excitation modes with lowest spin, supplemented by a tower of higher-spin fields. They arise from an internal structure which can be viewed as a twisted bundle over $S^{4}$, leading to a covariant noncommutative geometry. The linearized 4-dimensional Einstein equations are obtained from the classical matrix model action under certain conditions, modified by an IR cutoff. Some one-loop contributions to the effective action are computed using the formalism of string states.

KEYwords: M(atrix) Theories, Models of Quantum Gravity, Non-Commutative Geometry ARXIV EPRINT: 1606.00769 


\section{Contents}

1 Introduction 2

2 Covariant fuzzy four-spheres $\mathcal{S}_{\Lambda}^{4} \quad 4$

2.1 Semi-classical geometry and mode decomposition 5

2.2 Local description 8

$\begin{array}{lll}2.3 & \text { Functions versus symmetry generators } & 10\end{array}$

3 Matrix model and fluctuations on fuzzy $\mathcal{S}_{\Lambda}^{4} \quad 11$

3.1 Decomposition into fluctuation modes 13

$\begin{array}{ll}3.2 \text { Gauge transformation } & 14\end{array}$

4 Geometry: metric and vielbein $\quad 17$

4.1 Thick spheres, extra dimensions and dimensional reduction 20

$\begin{array}{lll}4.2 & \text { Effective metric and scalar fields } & 20\end{array}$

$\begin{array}{ll}4.3 & \text { Flux and field strength } \\ \end{array}$

5 Gravity $\quad 22$

5.1 Classical action and equations of motion 22

$\begin{array}{ll}5.2 \text { Curvature and linearized Einstein equations } & 27\end{array}$

6 One-loop corrections from string states $\quad 29$

6.1 The 1-loop effective potential for the IKKT model 29

$\begin{array}{lll}6.2 & \text { Vacuum energy of } \mathcal{S}_{N}^{4} & 32\end{array}$

$\begin{array}{lll}6.3 \text { Fluctuations } & 33\end{array}$

6.4 Transversal fluctuations 34

6.5 Tangential fluctuations 34

$\begin{array}{lll}7 & \text { Conclusion and outlook } & 36\end{array}$

$\begin{array}{lll}\text { A The classical geometry of the 4-spheres } \mathcal{S}_{\Lambda}^{4} & 38\end{array}$

$\begin{array}{lll}\text { A.1 The basic sphere } \mathcal{S}_{N}^{4} & 38\end{array}$

$\begin{array}{lll}\text { A.2 The generalized sphere } \mathcal{S}_{\Lambda}^{4} & 40\end{array}$

$\begin{array}{ll}\text { B Some identities for fuzzy 4-spheres } & 41\end{array}$

C Background flux $\theta^{\mu \nu}(x, \xi)$ averaged over $S^{2}$

D Mixed Young projections and permutations $\quad 42$

E Evaluation of $D^{2} \quad 43$ 


\section{Introduction}

Matrix models such as the IIB or IKKT model [1] (cf. [2,3]) provide fascinating candidates for a quantum theory of fundamental interactions. Their most interesting feature is that geometry is not an input, but arises itself as a brane-type solution with dynamical "quantum" geometry. Fluctuations around such solutions lead to gauge fields and matter fields on the background. It is natural to expect that gravity, along with the other fundamental interactions, should emerge on suitable backgrounds in the low-energy, semi-classical regime. Remarkably, numerical evidence for the emergence of 3+1-dimensional space-time within the finite-dimensional IIB model was reported recently $[4,5]$.

At first sight, the relation of the IIB matrix model with string theory suggests that 4-dimensional gravity can arise only if target space is compactified. This would not only lead to the well-known issues with a vast landscape of possibilities, it would also require ad-hoc modifications or constraints ${ }^{1}$ of the matrix model, destroying much of its appeal and simplicity. With this motivation, there were ongoing efforts to understand possible mechanisms for gravity in this model based solely on the 4-dimensional, non-commutative (NC) physics of the branes rather than the 10-dimensional bulk gravity (which arises in the matrix model upon quantization) [7-10]. Although 4-dimensional NC gauge theory behaves indeed very much like a gravitational theory [8, 9, 11-13], the emerging gravity on basic branes seems to be different from usual gravity, and it was not possible to derive the Einstein equations up to now.

In this paper, we show that Einstein-like gravity can indeed arise on more sophisticated, covariant noncommutative branes in this model, at least in some regime. This is based solely on the classical matrix model dynamics for fluctuation modes on the background brane, and has nothing to do with IIB supergravity in the bulk. The internal structure of the quantum space is crucial for the mechanism. This background is a generalized 4dimensional fuzzy sphere $\mathcal{S}_{\Lambda}^{4}$, but most of the considerations should apply also to analogous spaces with Minkowski signature.

There are two crucial features of $\mathcal{S}_{\Lambda}^{4}$ which are essential here [14]. First, it has an internal bundle structure, which transforms non-trivially under local space(time) rotations. Each point on the local fiber corresponds to a particular choice of an antisymmetric tensor $\theta^{\mu \nu}$ on $S^{4}$. This tensor is averaged over the fiber, leading to a covariant noncommutative structure of the 4-dimensional space. The second crucial feature is the fact that $\theta^{\mu \nu}$ (complemented by $P^{\mu}$ ) is not central, but generate the local Euclidean isometry group including translations. Quantum spaces with these features will be denoted as covariant quantum spaces. This concept is actually very old and goes back to Snyder and his proposal [15] for a Lorentz-invariant noncommutative Minkowski space. Fuzzy $S^{4}$ is a compact and well-controlled Euclidean version of such a space. Due to the extra generators $\theta^{\mu \nu}$ and $\mathcal{P}^{\mu}$, the corresponding algebra (of "functions") is larger than what seems to be needed in field theory, hence this type of space was not very much appreciated.

\footnotetext{
${ }^{1}$ For example, the toroidal compactifications considered in [6] require an infinite number of degrees of freedom and cannot be imposed in the finite-dimensional matrix model.
} 
In contrast to most previous work on this type of spaces (cf. [16, 17] and references therein), we take serious these extra fluctuation modes. They can be understood as harmonics on the internal fiber, which - in contrast to Kaluza-Klein compactification transform non-trivially under the local isometry group. This leads to an infinite tower of higher-spin fields, truncated at $N$ for fuzzy $\mathcal{S}_{N}^{4}$. Among the lowest modes in this tower, we identify the metric fluctuation, a selfdual $\mathrm{SO}(4)$ connection as well as gauge fields. We then perform a fluctuation analysis in the semi-classical limit along the lines of $[7,8]$. The metric fluctuation $H_{\mu \nu}$ is a combination of a rank 2 tensor field $h_{\mu \nu}$ and the divergence $\partial^{\rho} A_{\mu \rho \nu}$ of a $\mathrm{SO}(4)$-valued gauge field. Their semi-classical equations of motion of the classical matrix model then lead to the (linearized) Einstein equations for $H_{\mu \nu}$, for wavelengths below some scale $L_{R}$. Above this length scale, gravity no longer applies. However, this requires a certain type of generalized fuzzy spheres $\mathcal{S}_{\Lambda}^{4}$, and we have assume dimensional reduction to 4 dimensions, ignoring propagation in the compact extra dimensions. Mechanisms to ensure this are suggested, but this needs to be addressed in future work. This issue could be avoided by a suitable self-dual modification of the matrix model action.

The present framework incorporates several aspects of previous work in this context. Averaging over the Poisson structure $\theta^{\mu \nu}$ was considered in the DFR approach to field theory on the Moyal-Weyl plane [18], in order to preserve Lorentz invariance. However, $\theta^{\mu \nu}$ was considered as central there, which kills gravity. The effective metric and the dynamics of $\mathrm{NC}$ branes in matrix models was analyzed in $[7,8]$, but the backgrounds under consideration were too simple. Finally, an interpretation of the matrices as covariant derivatives rather than position operators was proposed in [19]. This also leads to higher spin fields with some similarities to the present framework and even the Einstein equations in vacuum, however this doesn't work in the finite-dimensional model, and the proper coupling to matter was not established. Due to the $\mathrm{SO}(5)$ setup, there are also similarities with the MacDowell-Mansouri formulation of GR [20, 21], however the physics is different: the full $\mathrm{SO}(5)$ symmetry is manifest here, and there are additional degrees of freedom beyond the ones in GR. The present framework shares aspects with noncommutative $\mathrm{SO}(5)$ gauge theory approaches [22-24], but again this is not quite appropriate: the gauge group is actually much larger here, corresponding to (a quotient of) $\mathrm{U}(\mathfrak{s o}(5))$.

The reason for insisting on the IIB model is that the quantization is well-behaved, since the non-local UV/IR mixing is mild due to maximal SUSY (and leads to IIB supergravity). In section 6 , we compute the leading terms in the one-loop effective action for the lowest fluctuation modes on $\mathcal{S}_{N}^{4}$. This is possible due to recent progress for the quantization of field theory on fuzzy spaces based on string states [25]. We show that previous one-loop results can be reproduced efficiently in this formalism, and some (preliminary) computations suggest that the one-loop effects can be captured by a minor generalization of the classical action, preserving the mechanism for gravity.

This paper is written in a pedestrian way, to make everything explicit and avoid getting trapped in some formalism. Of course there should be a more structural approach, and many limitations of this paper - notably the restriction to the linearized regime are clearly inessential. Other open issues include the coupling of the conformal mode to scalar fields which seems odd (see section 4.2), the proper extension to the Minkowski case, 
the justification of dimensional reduction via fuzzy extra dimensions, and the coupling to fermions. These should be addressed in future work. Nevertheless the basic mechanism is compelling, and certainly provides a serious candidate for a quantum theory of gravity which behaves similar to GR in a suitable range.

\section{Covariant fuzzy four-spheres $\mathcal{S}_{\Lambda}^{4}$}

We consider covariant fuzzy four-spheres defined in terms of 5 hermitian matrices $X^{a}, a=$ $1, \ldots, 5$ acting on some finite-dimensional Hilbert space $\mathcal{H}$, which transform as vectors under $\mathrm{SO}(5)$

$$
\begin{aligned}
{\left[\mathcal{M}_{a b}, X_{c}\right] } & =i\left(\delta_{a c} X_{b}-\delta_{b c} X_{a}\right) \\
{\left[\mathcal{M}_{a b}, \mathcal{M}_{c d}\right] } & =i\left(\delta_{a c} \mathcal{M}_{b d}-\delta_{a d} \mathcal{M}_{b c}-\delta_{b c} \mathcal{M}_{a d}+\delta_{b d} \mathcal{M}_{a c}\right)
\end{aligned}
$$

Here the $\mathcal{M}^{a b}=-\mathcal{M}^{b a}$ for $1 \leq a \neq b \leq 5$ define a (not necessarily irreducible) representation of $\mathfrak{s o}(5)$ on $\mathcal{H}$. The radius

$$
X^{a} X_{a}=\mathcal{R}^{2}
$$

is a scalar operator of dimension $L^{2}$, and the commutator of the $X^{a}$ will be denoted by

$$
\left[X^{a}, X^{b}\right]=: i \Theta^{a b}
$$

Here and throughout this paper, indices are raised and lowered with $g_{a b}=\delta_{a b}$. This type of relations constitute a covariant quantum space.

The form of the algebra (2.1) suggests a particular realization of such fuzzy fourspheres, based on an irreducible representation (irrep) of $\mathfrak{s o}(6)$ as follows ${ }^{2}$

$$
X^{a}=r \mathcal{M}^{a 6}, \quad a=1, \ldots, 5, \quad \Theta^{a b}=r^{2} \mathcal{M}^{a b}
$$

Here $\mathcal{M}^{a b}, a=1, \ldots, 6$ define an irrep of $\mathfrak{s o}(6) \cong \mathfrak{s u}(4)$ on $\mathcal{H}$, and $r$ is a scale parameter of dimension $L$. Correspondingly, $\mathfrak{s o}(5) \subset \mathfrak{s o}(6)$ is embedded by restricting the indices of $\mathcal{M}^{a b}$ to $a, b=1, \ldots, 5$. We also note the following simple identity for such spheres

$$
\left\{X_{a}, \Theta^{a b}\right\}_{+}=\left[\mathcal{R}^{2}, X^{b}\right] \neq 0 \quad \text { in general. }
$$

This is the type of space under consideration in this paper. There are important differences depending on the representation $\mathcal{H}$ of $\mathfrak{s o}(6)$ :

The basic fuzzy 4-sphere $\mathcal{S}_{N}^{4}$. The simplest example is the "basic" fuzzy four-sphere $\mathcal{S}_{N}^{4}$ [27-29], which is obtained for the highest weight irrep $\mathcal{H}=\mathcal{H}_{\Lambda}$ of $\mathfrak{s o}(6)$ with $\Lambda=$ $(0,0, N)$, denoting highest weights by their Dynkin indices. This representation can be realized as totally symmetric tensor product $\mathcal{H}_{\Lambda} \cong\left(\mathbb{C}^{4}\right)^{\otimes_{S} N}$ of the 4-dimensional (spinor)

\footnotetext{
${ }^{2}$ This is similar to an observation of Yang [26] in the context of the Snyder's noncommutative space.
} 
representation of $\mathfrak{s o}(6)$, which happens to remain irreducible as a $\mathfrak{s o}(5) \subset \mathfrak{s o}(6)$ representation. In this particular case, the radius operator is proportional to the identity operator,

$$
X^{a} X_{a}=\mathcal{R}^{2}=r^{2} R_{N}^{2} \mathbb{1}, \quad R_{N}^{2}=\frac{1}{4} N(N+4)
$$

For this basic fuzzy sphere $\mathcal{S}_{N}^{4}$, the following useful formulae are established in appendix B

$$
\begin{aligned}
\left\{X_{a}, \mathcal{M}^{a b}\right\}_{+} & =0 \\
\frac{1}{2}\left\{\Theta^{a b}, \Theta^{a^{\prime} c}\right\}_{+} g_{a a^{\prime}} & =r^{2} \mathcal{R}^{2}\left(g^{b c}-\frac{1}{2 \mathcal{R}^{2}}\left\{X^{b}, X^{c}\right\}_{+}\right) \\
\epsilon^{i j k l m} X_{i} X_{j} X_{k} X_{l} X_{m} & =(N+2) r^{3} \mathcal{R}^{2}
\end{aligned}
$$

where $\{., .\}_{+}$denotes the anti-commutator. As explained in appendix A, this is the quantization of a 6-dimensional coadjoint orbits of $\mathrm{SO}(6)$ mapped to $S^{4} \hookrightarrow \mathbb{R}^{5}$ via the $x^{a} \sim X^{a}$.

Generalized fuzzy 4-spheres $\mathcal{S}_{\Lambda}^{4}$. More general fuzzy 4-spheres are obtained for $\mathfrak{s o}(6)$ irreps $\mathcal{H}_{\Lambda}$ with $\Lambda=\left(n_{1}, n_{2}, N\right)$. As explained in appendix $\mathrm{A}$, these arise as quantizations of generic coadjoint $\mathrm{SO}(6)$ orbits, and the semi-classical geometry is that of a "thick" 4-sphere embedded in $\mathbb{R}^{5}$. As long as $n_{1}, n_{2} \ll N$, the radius $\mathcal{R}^{2}=X_{a} X^{a}$ is non-trivial but with sharply peaked spectrum around $r^{2} R_{N}^{2}$, with

$$
\left[\mathcal{R}^{2}, X^{b}\right] \neq 0
$$

As explained in appendix $\mathrm{A}, \mathcal{S}_{\Lambda}^{4}$ can be understood as a $\mathrm{SU}(3)$ bundle over $\mathcal{S}_{N}^{4}$. The relation (2.7) is modified as

$$
\frac{1}{2}\left\{\Theta^{a b}, \Theta^{a^{\prime} c}\right\}_{+} g_{a a^{\prime}}=r^{2} \mathcal{R}^{2}\left(g^{b c}-\frac{1}{2 \mathcal{R}^{2}}\left\{X^{b}, X^{c}\right\}_{+}+t^{b c}\right)
$$

where $t^{a b}=O\left(\frac{n}{N}\right)$, see (B.5) and (A.20). This generalization will be essential for gravity.

\subsection{Semi-classical geometry and mode decomposition}

As usual for fuzzy or noncommutative spaces, the matrix algebra $\operatorname{End}(\mathcal{H})$ constitutes the noncommutative algebra of functions or fields on the (generalized) fuzzy 4-sphere. The realization of $\mathcal{M}^{a b}$ in terms of generators of $\mathfrak{s o}(6) \cong \mathfrak{s u}(4)$ also provides the proper geometrical interpretation. We recall the well-known fact that $\operatorname{End}\left(\mathcal{H}_{\Lambda}\right)$ can be naturally interpreted as quantized algebra of functions on the coadjoint orbit $\mathcal{O}[\Lambda]=\left\{g \cdot \Lambda \cdot g^{-1} ; g \in\right.$ $\mathrm{SU}(4)\}$ of $\mathfrak{s u}(4)$ through the weight $\Lambda$ (cf. [30]). The generators $\mathcal{M}^{a b}, a, b=1, \ldots, 6$ are quantized embedding functions

$$
\mathcal{M}^{a b} \sim m^{a b}: \quad \mathcal{O}[\Lambda] \hookrightarrow \mathbb{R}^{15} \cong \mathfrak{s u}(4)
$$

dual to some ON basis $\lambda^{a b}$ of $\mathfrak{s u}(4)$. In particular, the $X^{a} \sim \mathcal{M}^{a 6}$ are naturally interpreted as projections of such coadjoint orbits to $S^{4} \subset \mathbb{R}^{5}$,

$$
X^{a} \sim x^{a}: \quad \mathcal{O}[\Lambda] \hookrightarrow \mathbb{R}^{15} \stackrel{\Pi}{\rightarrow} S^{4} \subset \mathbb{R}^{5}
$$


where $\Pi$ denotes the projection of $\mathfrak{s u}(4)$ to the subspace spanned by the $\lambda^{a 6}$ generators. Hence the fuzzy 4-spheres are actually higher-dimensional homogeneous spaces which are twisted bundles over $S^{4}$, with the fiber playing the role of a hidden extra dimension. In contrast to standard Kaluza-Klein compactifications, these extra dimensions lead to higherspin modes here. For the basic 4-sphere $\mathcal{S}_{N}^{4}$, the underlying orbit is $\mathcal{O}[\Lambda]=\mathbb{C} P^{3}$, which is a $S^{2}$ bundle over $S^{4}$ as elaborated in [14,31-36]. More details on the geometry including the generic case are given in appendix A. In particular, the space of classical functions on these orbits is spanned by polynomials $F\left(x^{a}, m^{a b}\right)$, which are in one-to-one correspondence with the noncommutative modes (2.26), up to some UV cutoff defined by $N$ and $n_{i}$.

Poisson structure. This geometrical picture also explains the origin of the commutator as quantized (Kirillov-Kostant) Poisson bracket on $\mathcal{O}[\Lambda]$. This Poisson structure can be viewed as a 2 -vector field on $\mathcal{O}[\Lambda]$

$$
\{f, g\}=\theta^{A B} \partial_{A} f \partial_{B} g
$$

whose projection (push-forward) to $S^{4}$ is given by

$$
\theta^{\mu \nu}(x, \xi) \partial_{\mu} \otimes \partial_{\nu}
$$

Here $\xi$ are coordinates on the internal fiber of $\mathcal{O}[\Lambda]$ over $S^{4}$. For the basic 4 -sphere $\mathcal{S}_{N}^{4}$, $\theta^{\mu \nu}(x, \xi)$ is selfdual (SD) at each $(x, \xi)$, defining a bundle of SD frames over $S^{4}$, which rotates (and averages out) along the fiber $S^{2}$. More precisely, it transforms as $(1,0)$ under the local $\mathrm{SO}(4)=\mathrm{SU}(2)_{L} \otimes \mathrm{SU}(2)_{R}$ rotations, which are implemented by the action of $\left\{\theta^{\mu \nu},.\right\}$ on itself. In the noncommutative case, this amounts to a gauge transformation

$$
\Lambda^{\mu \mu^{\prime}} \Lambda^{\nu \nu^{\prime}} \Theta^{\mu^{\prime} \nu^{\prime}}=U_{\Lambda} \Theta^{\mu \nu} U_{\Lambda}^{-1} .
$$

In other words, local rotations are implemented as gauge transformations, which already hints towards gravity.

For the 5 embedding functions $x^{a} \sim X^{a}$, the Poisson bracket

$$
\left\{x^{a}, x^{b}\right\}=\theta^{a b}: \quad \mathcal{O}[\Lambda] \hookrightarrow \mathfrak{s o}(5) \subset \mathfrak{s o}(6)
$$

gives rise to $\Theta^{a b}$ is the fuzzy case. Once again, $\theta^{a b}$ is only defined on the bundle $\mathcal{O}[\Lambda]$, it is not a Poisson bracket on $S^{4}$, since it is not constant along the fiber. ${ }^{3}$

Much of the analysis in this paper is done in this semi-classical limit indicated by $\sim$, replacing commutators by Poisson brackets and working with $\mathcal{O}[\Lambda]$. This greatly simplifies the analysis, and it is certainly justified in the gravity regime where the typical wavelengths are much longer than the scale of noncommutativity.

Coherent states. As for all quantized coadjoint orbits, coherent states on $\mathcal{O}[\Lambda]$ are given by highest weight states $|\Lambda\rangle \in \mathcal{H}_{\Lambda}$ and their $\mathrm{SO}(6)$ orbits,

$$
\begin{aligned}
|\mathbf{x}\rangle & \equiv|x ; \xi\rangle=g_{\mathbf{x}} \cdot|\Lambda\rangle, \quad g_{\mathbf{x}} \in \mathrm{SO}(6) \\
x^{a} & =\left\langle\mathbf{x}\left|X^{a}\right| \mathbf{x}\right\rangle \equiv\left\langle X^{a}\right\rangle .
\end{aligned}
$$

\footnotetext{
${ }^{3}$ Recall that $H^{2}\left(S^{4}\right)=0$, hence there is no symplectic 2-form on $S^{4}$.
} 
Up to a $\mathrm{U}(1)$ phase factor, they are in one-to-one correspondence to points $\mathbf{x}$ on $\mathcal{O}[\Lambda]$. Alternatively, we can use the $\mathrm{SO}(5)$ point of view and consider highest weight states of the $\mathrm{SO}(5)$ modules. It will suffice here to consider the case of the basic fuzzy sphere $\mathcal{S}_{N}^{4}$, where both notions coincide. We can then label the points on the bundle $\mathcal{O}[\Lambda] \cong \mathbb{C} P^{3}$ locally by $x \in S^{4}$ and $\xi$, where the "north pole" $\mathbf{p}$ corresponds to the highest weight state $|\Lambda\rangle$. They are optimally localized, minimizing the uncertainty in position space ${ }^{4}$

$$
\begin{aligned}
\Delta^{2} & :=\sum_{a}\left\langle\left(X^{a}-\left\langle X^{a}\right\rangle\right)^{2}\right\rangle=\sum_{a}\left\langle\left(X^{a}\right)^{2}\right\rangle-\left\langle X^{a}\right\rangle^{2} \\
& =\left(R_{N}^{2}-\frac{1}{4} N^{2}\right) r^{2} \sim \frac{4}{N} \mathcal{R}^{2}=2 r \mathcal{R} \\
& =: L_{N C}^{2}
\end{aligned}
$$

which defines the length scale $L_{N C}$. One can then associate to any operator $\phi \in \operatorname{End}(\mathcal{H})$ a function $\phi(\mathbf{x})$ on $\mathcal{O}[\Lambda]$ as follows

$$
\phi(\mathbf{x})=\langle\mathbf{x}|\phi| \mathbf{x}\rangle
$$

and the semi-classical regime is characterized by functions $\phi(x)$ which vary on scales $>L_{N C}$. The coherent states form a $\mathrm{U}(1)$ bundle over $\mathcal{O}[\Lambda]$, with a canonical connection whose curvature gives the symplectic form $\omega$ on $\mathcal{O}[\Lambda]$, corresponding to the Poisson structure

$$
i \theta^{a b}(\mathbf{x})=\left\langle\mathbf{x}\left|\left[X^{a}, X^{b}\right]\right| \mathbf{x}\right\rangle .
$$

This also encodes the uncertainty scale $L_{N C}$ and the volume quantization via (2.8). Finally, the trace over $\operatorname{End}(\mathcal{H})$ can be realized by the integral over all coherent states on $\mathcal{O}[\Lambda]$,

$$
\operatorname{Tr} \phi=\frac{\operatorname{dim} \mathcal{H}_{\Lambda}}{\operatorname{Vol} \mathcal{O}[\Lambda]} \int_{\mathcal{O}} d \mathbf{x}\langle\mathbf{x}|\phi| \mathbf{x}\rangle .
$$

This locally separates into an integration over $S^{4}$ times the internal fiber $\mathcal{F}$, which allows to evaluate the matrix model actions in a standard semi-classical form.

Scalar fields and higher-spin modes. The most general functions on fuzzy $\mathcal{S}_{N}^{4}$ are organized into the following $\mathrm{SO}(6)$ resp. $\mathrm{SO}(5)$ modes (cf. [29, 31, 35, 36])

$$
\phi \in \operatorname{End}(\mathcal{H}) \cong \bigoplus_{n \leq N}(n, 0, n)_{\mathfrak{s o}(6)} \cong \bigoplus_{m \leq n \leq N}(n-m, 2 m)_{\mathfrak{s o}(5)},
$$

denoting highest weight irreducible representations (irreps) by their Dynkin indices; for example, $(n, m)$ denotes the $\mathfrak{s o}(5)$ irrep with highest weight $\Lambda=n \Lambda_{1}+m \Lambda_{2}$ where $\alpha_{1}$ is the long root and $\alpha_{2}$ the short root. We are mainly interested in the "low spin" representations with small $m$. Then a more explicit realization is obtained in terms of ordered polynomials ${ }^{5}$ in the generators $X^{a}$ and $\mathcal{M}^{a b}, a, b=1, \ldots, 5$. For example, scalar fields on $S^{4}$ correspond

\footnotetext{
${ }^{4}$ Here $\left\langle\mathbf{p}\left|\left(X^{5}\right)^{2}\right| \mathbf{p}\right\rangle=\frac{r^{2}}{4} N^{2}$ at the north pole $p$ follows using the explicit realization of $X^{a}$ in terms of gamma matrices [28].

${ }^{5}$ This is a quotient of the Poincare-Birkhoff-Witt basis of $\mathrm{U}(\mathfrak{s o}(6))$.
} 
to the $(n, 0)$ modes, realized by totally symmetric polynomials $F(X)=F_{a_{1} \ldots a_{n}} X^{a_{1}} \ldots X^{a_{n}}$, and denoted by

$$
\mathcal{C}_{N}\left(S^{4}\right):=\bigoplus_{n \leq N} F_{n}(X) \cong \bigoplus_{n \leq N}(n, 0)
$$

Then

$$
\phi(\mathbf{x})=\langle\mathbf{x}|\phi| \mathbf{x}\rangle, \quad \phi \in \mathcal{C}_{N}\left(S^{4}\right)
$$

is constant along the fiber and defines a function on $S^{4}$. There is an associated projection map [29]

$$
\phi \mapsto[\phi]_{0}:=\phi_{0} \in \mathcal{C}_{N}\left(S^{4}\right),
$$

which picks out the scalar modes $(n, 0)$ in $(2.22)$. In the semi-classical limit, this corresponds to integrating $\phi(\mathbf{x})$ over the internal fiber.

More generally, we can organize all other higher spin fields in terms of polynomials with "internal" generators $\mathcal{M}^{a b}, a, b=1, \ldots, 5$ multiplied by scalar functions. For example,

$$
\begin{aligned}
F_{a b}(X) \mathcal{M}^{b c} & =F_{a_{1} \ldots a_{n} ; b c} X^{a_{1}} \ldots X^{a_{n}} \mathcal{M}^{b c} \in(n+1,2)_{\mathfrak{s o}(5)} \\
F_{b c ; d e}(X) \mathcal{M}^{b c} \mathcal{M}^{d e} & =F_{a_{1} \ldots a_{n} ; b c ; d e} X^{a_{1}} \ldots X^{a_{n}} \mathcal{M}^{b c} \mathcal{M}^{d e} \in(n+2,4)_{\mathfrak{s o}(5)}
\end{aligned}
$$

and so forth, where the $F_{a_{1} \ldots a_{n} ; b c}$ and $F_{a_{1} \ldots a_{n} ; b c ; d e}$ are tensors of $\mathrm{SO}(5)$ corresponding to Young tableaux with one row of length 2 and two rows of length 2 , respectively. In particular, the $F_{b c}(X) \mathcal{M}^{b c}$ can be identified with 2 -forms $F_{b c}(x) d x^{b} \wedge d x^{c}$ on $\mathbb{R}^{5}$. These $(n, m)$ modes with $m \neq 0$ correspond to functions on $\mathcal{O}[\Lambda] \cong \mathbb{C} P^{3}$ which are non-trivial harmonics on the $S^{2}$ fiber. They are higher-spin fields on $S^{4}$ rather than Kaluza-Klein modes, because the local Lorentz group acts non-trivially on the internal $S^{2}$ fiber. This leads to a higher-spin theory, and we will show that its spin 2 sector describes gravity, but only for the generic spheres $\mathcal{S}_{\Lambda}^{4}$.

For the generalized spheres $\mathcal{S}_{\Lambda}^{4}$, the scalar operator $\mathcal{R}^{2}=X_{a} X^{a}$ is a non-trivial $\mathfrak{s o}(5)$ Casimir operator which distinguishes some of the internal structure. Then the mode decomposition is analogous but more complicated, with multiplicities arising in the decomposition (2.22). E.g. for $\Lambda=(k, 0, N)$, one finds schematically

$$
\operatorname{End}\left(\mathcal{H}_{\Lambda}\right) \cong \bigoplus_{n \leq N} k_{n}(n, 0, n) \oplus \ldots \text { (other modes) }
$$

\subsection{Local description}

We would like to understand the local structure from a field theory point of view, near some reference point $p \in S^{4}$ denoted as "north pole". We pick a coherent state $|\mathbf{p}\rangle$ to mark this point. Throughout this paper, tensorial objects at the (arbitrary) point $p \in S^{4}$ will be expressed in terms of the local tangent space $T_{p} S^{4}$, using the 4 tangential Cartesian coordinates $x^{\mu}$ centered at $p$, with

$$
x^{\mu}(p)=\left\langle\mathbf{p}\left|X^{\mu}\right| \mathbf{p}\right\rangle=0, \quad\left\langle\mathbf{p}\left|X^{5}\right| \mathbf{p}\right\rangle=\frac{r N}{2}=: R \approx \mathcal{R}
$$

assuming $n_{i} \ll N$. Then quantities such as $x^{\mu}(p)$ can always be dropped, greatly simplifying the analysis. Thus we can view $x^{\mu}$ as Riemannian normal coordinates at $p$ with respect 
to the embedding metric $g_{\mu \nu}$ of $S^{4} \subset \mathbb{R}^{5}$, and $\left.\nabla_{\mu}^{[g]}\right|_{p}=\left.\partial_{\mu}\right|_{p}$. To avoid confusions with the effective gravitational metric, we will use the symbol $\partial_{\mu}$ for $\nabla_{\mu}^{[g]}$, and we will often drop its ("cosmologically small") curvature $\left[\nabla_{\mu}^{[g]}, \nabla_{\nu}^{[g]}\right]=O\left(\frac{1}{R^{2}}\right)$ for simplicity. The generators separate accordingly as

$$
X^{a}=\left(\begin{array}{c}
X^{\mu} \\
X^{5}
\end{array}\right)
$$

with $X^{5}=\sqrt{R^{2}-X_{\mu} X^{\mu}}$, and the 4 matrices $X^{\mu} \sim x^{\mu}$ are quantizations of these local coordinates. The stabilizer of $p\left(\right.$ or $\left.X^{5}\right)$ is given by $\mathrm{SO}(4)$. Accordingly, $\mathfrak{s o}(5)$ decomposes into $\mathfrak{s o}(4)$ and local "translation" generators,

$$
\mathcal{M}^{a b}=\left(\begin{array}{cc}
\mathcal{M}^{\mu \nu} & \mathcal{P}^{\mu} \\
-\mathcal{P}^{\mu} & 0
\end{array}\right) \quad \text { where } \quad \mathcal{P}^{\mu}=\mathcal{M}^{\mu 5}
$$

In this setup, the (Euclidean) Poincare-group $I \mathrm{SO}(4)$ is recovered as usual by a contraction

$$
P_{\mu}=\frac{1}{R} g_{\mu \nu} \mathcal{P}^{\nu}, \quad X^{\mu}=r Z^{\mu}, \quad R=R_{N} r
$$

taking $R$ to be much larger than any other length scale under consideration. Then the $\mathcal{S}_{\Lambda}^{4}$ algebra takes the form

$$
\begin{aligned}
{\left[P_{\mu}, X^{\nu}\right] } & =i \frac{X^{5}}{R} \delta_{\mu}^{\nu} \quad \stackrel{R \rightarrow \infty}{\rightarrow} i \delta_{\mu}^{\nu}, \\
{\left[P_{\mu}, P_{\nu}\right] } & =\frac{i}{R^{2}} \mathcal{M}^{\mu \nu} \quad \stackrel{R \rightarrow \infty}{\rightarrow} 0 \\
{\left[X^{\mu}, X^{\nu}\right] } & =: i \theta^{\mu \nu}=i r^{2} \mathcal{M}^{\mu \nu}
\end{aligned}
$$

assuming $r \ll 1$. Here and in the following, greek indices indicate that the corresponding tensor is tangential.

Poisson algebra limit. Now consider again the semi-classical (Poisson) limit. The exact relations (2.7) for $\mathcal{S}_{N}^{4}$ then imply the following important formula

$$
\begin{aligned}
g_{c c^{\prime}} \theta^{c a} \theta^{c^{\prime} b} & =\frac{1}{4} \Delta^{4} P_{T}^{a b}, & P_{T}^{a b} & =g^{a b}-\frac{1}{R^{2}} x^{a} x^{b}, \\
P_{T}^{2} & =P_{T}, & P_{T} \cdot x & =0 .
\end{aligned}
$$

where $P_{T}^{a b}$ is the projector on the tangent space of $S^{4} \subset \mathbb{R}^{5}$. This allows to evaluate the kinetic term of a scalar field $\phi \in \mathcal{C}_{N}\left(S^{4}\right)(2.23)$ in the semi-classical limit:

$$
-g_{a b}\left[X^{a}, \phi\right]\left[X^{b}, \phi\right] \sim g_{a b} \theta^{a \mu^{\prime}} \theta^{b \nu^{\prime}} \partial_{\mu^{\prime}} \phi \partial_{\nu^{\prime}} \phi=\gamma^{\mu \nu} \partial_{\mu} \phi \partial_{\nu} \phi
$$

As always, this is obtained by replacing commutators with Poisson brackets. Here

$$
\gamma^{\mu \nu}:=g_{a b} \theta^{a \mu} \theta^{b \nu}=\frac{1}{4} \Delta^{4} g^{\mu \nu}
$$


will play a prominent role as effective background metric. In contrast to $\theta^{\mu \nu}$, this is indeed a tensor on $S^{4}$, i.e. it is constant along the fiber. For the translation generators $p_{\mu}=\frac{1}{R r^{2}} \theta^{\mu 5}$, equation (2.8) implies

$$
\theta^{\mu \nu} p_{\nu}=\frac{1}{R r^{2}} \theta^{\mu \nu} \theta_{\nu 5}=\frac{x^{5}}{R} x^{\mu}
$$

This shows that on $\mathcal{S}_{N}^{4}$, the functions $p_{\mu}$ are not independent and basically vanish, since $x_{\mu}=0$ in the local frame at any given point $p \in S^{4}$. See appendix A for more details.

In contrast for the generic spheres $\mathcal{S}_{\Lambda}^{4}$, the $p_{\nu}$ are independent functions, and (2.33) is replaced by

$$
g_{c c^{\prime}} \theta^{c a} \theta^{c^{\prime} b}=\frac{1}{4} \Delta^{4}\left(P_{T}^{a b}+t^{a b}\right)=: \gamma^{a b}
$$

(see (A.20) in appendix A) where $t^{a b}$ has a non-vanishing radial component. Upon averaging over the local fiber, this defines a scale

$$
\left[\theta^{\mu 5} \theta^{\nu 5}\right]_{0}=: L_{R}^{2} r^{2} g^{\mu \nu}, \quad L_{R}^{2}=r^{2}\left(c_{n}^{2}+N\right) \geq \Delta^{2} .
$$

$L_{R}$ characterizes the thickness of the sphere $\mathcal{S}_{\Lambda}^{4}$ with $\Lambda=\left(n_{1}, n_{2}, N\right)$, and the contribution $N$ in (2.38) arises from the uncertainty ${ }^{6}$ of $X^{\mu}$ in (2.8). In particular, this gives

$$
\left[p_{\mu} p_{\nu}\right]_{0}=\frac{4 L_{R}^{2}}{\Delta^{4}} g_{\mu \nu}
$$

and

$$
\frac{\left|\theta^{\mu 5}\right|}{\left|\theta^{\mu \nu}\right|}=\frac{L_{R}}{R}=O\left(\frac{\sqrt{N+n^{2}}}{N}\right) .
$$

\subsection{Functions versus symmetry generators}

It is important to keep in mind the double meaning of the generators $\theta^{\mu \nu}$ and $P_{\mu}$ :

1. as symmetry generators of the isometry group, which act on wavefunctions via the adjoint. Then the normalization $\mathcal{M}^{\mu \nu}=r^{-2} \Theta^{\mu \nu}$ is appropriate. In particular $P_{\mu}$ and $\mathcal{M}^{\mu \nu}$ generate the local Poincare algebra for $R \rightarrow \infty$.

2. as generators of the algebra of functions on $\mathcal{O}[\Lambda]$ (along with $x^{\mu}$ ), viewed as bundles over $S^{4}$. In the fuzzy case, this is replaced by $\operatorname{End}\left(\mathcal{H}_{\Lambda}\right)$, which describes the degrees of freedom in a field theory (or matrix model) on $\mathcal{S}_{\Lambda}^{4}$. This algebra is "almost" commutative for large $\Lambda$.

Consider e.g. the basic sphere $\mathcal{S}_{N}^{4}$. Since the underlying space $\mathcal{O}[\Lambda] \cong \mathbb{C} P^{3}$ is 6 dimensional, there are locally only 6 independent coordinate functions. At the north pole, these are the $x^{\mu}$, plus 2 of the 3 selfdual $\theta^{\mu \nu}$ variables which parametrize the fiber $S^{2}$. The $\theta^{\mu \nu}$ can be viewed as function on $\mathbb{C} P^{3}$ taking values in the SD 2-forms $\Omega^{2}\left(S^{4}\right)$ or in the local $\mathfrak{s u}(2)_{L} \subset \mathfrak{s o}(4)$. However, the $p^{\mu}$ functions vanish in the semi-classical limit, as

\footnotetext{
${ }^{6}$ This can also be seen using e.g. the explicit representation of $\mathcal{S}_{N}^{4}$ on $\mathcal{H}=(0, N)$. Similar effects are well-known for $S_{N}^{2}$.
} 
explained above. Therefore there are no modes of the type $F_{\mu}(X) P^{\mu}$ on $\mathcal{S}_{N}^{4}$, but such modes do exist on the generic spheres $\mathcal{S}_{\Lambda}^{4}$; this will be crucial below.

Now consider the symmetry generators and their action on wavefunctions. The quadratic Casimir of $\mathrm{SO}(5)$ can be written as

$$
\begin{aligned}
C^{2}[\mathfrak{s o}(5)] & =\sum_{a<b \leq 5} \mathcal{M}_{a b} \mathcal{M}_{a b}=\sum_{\mu<\nu \leq 4} \mathcal{M}_{\mu \nu} \mathcal{M}^{\mu \nu}+\sum_{\mu} \mathcal{P}_{\mu} \mathcal{P}^{\mu} \\
& =R^{2}\left(P_{\mu} P^{\mu}+\frac{1}{R^{2}} \mathcal{M}_{\mu \nu} \mathcal{M}^{\mu \nu}\right)
\end{aligned}
$$

using the same symbols for the abstract generators as in the $\mathcal{S}_{N}^{4}$ algebra. Acting on scalar fields (or on fields with low spin), the angular momentum contribution can be neglected ${ }^{7}$ compared with the translational contribution, $C^{2}[\mathfrak{s o}(5)] \approx R^{2} P^{\mu} P_{\mu}$. Comparing the formula for its eigenvalues

$$
C^{2}[\mathfrak{s o}(5)](n-m, 2 m)=n(n+3)+m(m+1)
$$

with the formula for the eigenvalues of $\square=\left[X^{a},\left[X_{a},.\right]\right]=C^{2}[\mathfrak{s o}(6)]-C^{2}[\mathfrak{s o}(5)]$ on $\mathcal{S}_{N}^{4}[36]$

$$
\square(n-m, 2 m)=r^{2}(n(n+3)-m(m+1)), \quad m \leq n,
$$

it follows that

$$
\square \approx r^{2} C^{2}[\mathfrak{s o}(5)] \mid \approx-\frac{\Delta^{4}}{4} P^{\mu} P_{\mu}=-\frac{\Delta^{4}}{4} \square_{g}, \quad \square_{g}:=g^{\mu \nu} \partial_{\mu} \partial_{\nu}
$$

for the low-spin modes $m=0,1,2$. A simpler way to understand this is via the semiclassical form of the free action for scalar fields [8]

$$
\begin{aligned}
\operatorname{Tr} f \square g & \sim \int-\Omega f(x)\left\{x^{a},\left\{x_{a}, g(x)\right\}\right\}=\int \Omega\left\{x^{a}, f(x)\right\}\left\{x_{a}, g(x)\right\} \\
& =\int \Omega \gamma^{\mu \nu} \partial_{\mu} f \partial_{\nu} g=-\int \Omega f \gamma^{\mu \nu} \partial_{\mu} \partial_{\nu} g \sim-\operatorname{Tr} f\left(\gamma^{\mu \nu} \partial_{\mu} \partial_{\nu}\right) g
\end{aligned}
$$

where $\Omega$ is the symplectic volume form, in agreement with (2.44). This shows again that $\gamma^{\mu \nu}(2.35)$ is the effective metric on $\mathcal{S}_{\Lambda}^{4}$.

\section{$3 \quad$ Matrix model and fluctuations on fuzzy $\mathcal{S}_{\Lambda}^{4}$}

Now we would like to make $\mathcal{S}_{\Lambda}^{4}$ dynamical, by considering as a background in the Yang-Mills matrix model

$$
S[Y]=\frac{1}{g^{2}} \operatorname{Tr}\left(-\left[Y_{a}, Y_{b}\right]\left[Y^{a}, Y^{b}\right]+\mu^{2} Y^{a} Y_{a}\right)
$$

with a mass term as a regulator, and studying the fluctuation modes on $\mathcal{S}_{\Lambda}^{4}$. We will later focus on the IKKT model [1] with $D=10$. The classical equations of motion are

$$
\left(\square+\frac{1}{2} \mu^{2}\right) Y_{a}=0, \quad \square=\left[Y^{a},\left[Y_{a}, .\right]\right] .
$$

\footnotetext{
${ }^{7}$ Remember that we always work in the local frame at $p$, where any $x^{\mu}$ can be dropped.
} 
We use the letter $Y$ to indicate generic configurations, while $X$ will indicate the fuzzy $\mathcal{S}_{\Lambda}^{4}$ background. Although the latter is not quite a solution of these equations, it was shown in [36] that quantum corrections (at one loop) can stabilize the radius of $\mathcal{S}_{\Lambda}^{4}$ for small positive $\mu^{2}$. A more refined one-loop analysis will be presented in section 6 . Now consider fluctuations around some fixed background $X^{a}$,

$$
Y^{a}=X^{a}+\mathcal{A}^{a}
$$

where $\mathcal{A}^{a} \in \operatorname{End}(\mathcal{H})$ will be the dynamical degrees of freedom. Expanding the action expanded up to second oder in the $\mathcal{A}^{a}$, one obtains

$$
S[Y]=S[X]+\frac{2}{g^{2}} \operatorname{Tr}\left(2 \mathcal{A}^{a}\left(\square+\frac{1}{2} \mu^{2}\right) X_{a}+\mathcal{A}_{a}\left(\square+\frac{1}{2} \mu^{2}\right) \mathcal{A}_{a}-2\left[\mathcal{A}_{a}, \mathcal{A}_{b}\right]\left[X^{a}, X^{b}\right]-f^{2}\right) .
$$

dropping the linear terms (for stable backgrounds). Hence the quadratic fluctuations $\mathcal{A}^{a}$ are governed by the quadratic form

$$
\operatorname{Tr} \mathcal{A}_{a}\left(\left(\square+\frac{1}{2} \mu^{2}\right) \delta_{b}^{a}+2 i\left[\Theta^{a b}, .\right]\right) \mathcal{A}_{b}
$$

where the $f^{2}$ term was canceled by adding a suitable Faddeev-Popov gauge-fixing term (choosing the Feynman gauge [37]) for the gauge fixing function

$$
f=i\left[\mathcal{A}^{a}, X_{a}\right]
$$

Hence the fluctuations are governed by the "vector" (matrix) Laplacian

$$
\left(D^{2} \mathcal{A}\right)_{a}:=\left(\square+\frac{1}{2} \mu^{2}-M_{r s}^{(\mathcal{A})}\left[\Theta^{r s}, .\right]\right)_{b}^{a} \mathcal{A}_{b}
$$

where

$$
\left(M_{a b}^{(\mathcal{A})}\right)_{d}^{c}=i\left(\delta_{b}^{c} \delta_{a d}-\delta_{a}^{c} \delta_{b d}\right)
$$

is the $\mathrm{SO}(5)$ generator in the vector representation. The fluctuations $\mathcal{A}^{a}$ entail fluctuations of the "flux"

$$
\begin{aligned}
-i\left[Y^{a}, Y^{b}\right]=\Theta_{(Y)}^{a b} & =\Theta_{(X)}^{a b}+\mathcal{F}^{a b}, \\
\mathcal{F}^{a b} & =-i\left[X^{a}, \mathcal{A}^{b}\right]+i\left[X^{b}, \mathcal{A}^{a}\right]-i\left[\mathcal{A}^{a}, \mathcal{A}^{b}\right] .
\end{aligned}
$$

For backgrounds given by basic noncommutative branes $\mathcal{M}$, this leads to noncommutative gauge theory, or equivalently to a theory of geometric deformations

$$
y^{a}: \quad \mathcal{M} \hookrightarrow \mathbb{R}^{10}
$$

leading to some emergent gravity on the brane which seems to be different from general relativity $[8,38]$. However on the covariant $\mathcal{S}_{\Lambda}^{4}$ backgrounds, we will argue that (at least linearized) general relativity arises indeed from certain deformation modes, extended by a higher spin sector. 


\subsection{Decomposition into fluctuation modes}

Global SO(5) notation. Given some deformation $X^{a}+\mathcal{A}^{a}$ of the $S_{\Lambda}^{4}$ background, we want to identify the various fluctuation modes of the 5 fields $\mathcal{A}^{a}$. We can organize the tangential and radial fluctuations as follows, working mostly in the semi-classical limit

$$
\mathcal{A}^{a}=\xi^{a}+\theta^{a b} \mathbf{A}_{b}+\frac{X^{a}}{R} \kappa .
$$

Here

$$
\begin{aligned}
\xi^{a} & =\xi^{a}+\xi^{a b c} \mathcal{M}_{b c}+\ldots, & X_{a} \xi^{a} & =0 \\
\mathbf{A}_{a} & =A_{b}+A_{b c d} \mathcal{M}^{c d}+\ldots, & X^{a} \mathcal{A}_{a} & =0 \\
\kappa & =\kappa+\kappa_{a b} \mathcal{M}^{a b}+\ldots & &
\end{aligned}
$$

and the functions $\xi^{a}, A_{b}, A_{a b c}, \kappa \in \mathcal{C}_{N}\left(S^{4}\right) \subset \operatorname{End}(\mathcal{H})$ play the role of tensor fields. The expansion in $\mathcal{M}$ correspond to expanding $\operatorname{End}(\mathcal{H})=\oplus(n, 2 m)$ in terms of $m$. The $\xi^{a}$ and the $\mathbf{A}_{a}$ are clearly tangential, and $\kappa$ describes the radial fluctuations. We will only keep tensors of rank up to 3 . The $\mathbf{A}_{a}$ contribution is reminiscent of the standard parametrization in noncommutative gauge theory, and could be interpreted as $\mathfrak{u}(1) \times \mathfrak{s o}(5)$-valued gauge field (or more generally as $\mathrm{U}(\mathfrak{s o}(5))$-valued gauge field). Since the $X^{a}$ and $\mathcal{M}^{a b}$ are tensor operators, there is an $\mathrm{SO}(5)$ action on these fields via

$$
\mathbf{A}_{a} \rightarrow \Lambda_{a}^{b} U_{\Lambda} \mathbf{A}_{b} U_{\Lambda}^{-1}, \quad \kappa \rightarrow U_{\Lambda} \kappa U_{\Lambda}^{-1}
$$

etc., which leaves the background sphere invariant and implements the isometries on the tensor modes. In this sense, the theory to be elaborated will be "covariant". The extension to local gauge symmetries will be discussed shortly.

Now observe that the trace sector of $A_{b c d}$

$$
A_{b c d}=\frac{1}{2 \mathcal{R}^{2}}\left(g_{b d} \tilde{\xi}_{c}-g_{b c} \tilde{\xi}_{d}\right)
$$

leads to

$$
\mathcal{A}^{a}=\theta^{a b} A_{b c d} \mathcal{M}^{c d}=\left(P_{T} \tilde{\xi}\right)^{a}
$$

using (2.33), which is redundant with the $\xi^{a}$ modes. Therefore we should either impose that $A_{b c d}$ is traceless, or drop the $\xi^{a}$ modes (and the $\kappa$ modes for the generic spheres $\mathcal{S}_{\Lambda}^{4}$ ). We will mostly choose the latter option.

Local SO(4) notation. To make the physical content more transparent, we will organize these fields further into 4 D fields near some reference point $p \in S^{4}$ ("the north pole"). We will use greek indices $\mu, \nu \in\{1, \ldots, 4\}$ for tangential components transforming as vectors under the local $\mathrm{SO}(4)$ around $p \in S^{4}$, and latin indices $a, b, \ldots \in\{1, \ldots, 5\}$ for the $\mathrm{SO}(5)$ covariant components. In particular, the fields will be locally expanded in powers of the $\mathrm{SO}(4)$-covariant generators as in section 2.3 ,

$$
\Theta^{\mu \nu}=r^{2} \mathcal{M}^{\mu \nu} \quad \text { and } \quad P^{\mu}:=\frac{1}{R} \mathcal{M}^{\mu 5} .
$$




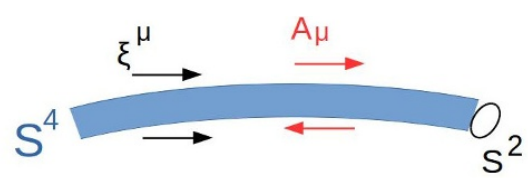

Figure 1. Schematic local picture of the deformation modes $A_{\mu}$ and $\xi^{\mu}$.

This organization gives the following modes up to the order under consideration here:

$$
\mathcal{A}^{\mu}=\xi^{\mu}+\frac{x^{\mu}}{R} \kappa+\theta^{\mu \nu} \mathbf{A}_{\nu}, \quad \mathcal{A}^{5}=\kappa
$$

where

$$
\begin{aligned}
\mathbf{A}_{\nu} & =A_{\nu}+A_{\nu \rho} P^{\rho}+A_{\nu \rho \sigma} \mathcal{M}^{\rho \sigma}+\ldots \\
\xi^{\mu} & =\xi^{\mu}(x)+\xi^{\mu \nu}(x) P_{\nu}+\xi^{\mu \nu \rho}(x) \mathcal{M}_{\nu \rho}+\ldots \\
\kappa & =\kappa+\kappa_{\mu} P^{\mu}+\kappa_{\mu \nu} \mathcal{M}^{\mu \nu}+\ldots
\end{aligned}
$$

where both $A_{\nu \rho \sigma}$ and $A_{\nu \rho}=A_{\nu \rho 5}$ arise form the $5 \mathrm{D}$ fields $A_{a b c}$. We separate $A_{\mu \nu}$ into symmetric and antisymmetric (AS) parts,

$$
A_{\nu \rho}=\frac{1}{2}\left(h_{\nu \rho}+a_{\nu \rho}\right), \quad h_{\nu \rho}=h_{\rho \nu}, \quad a_{\nu \rho}=-a_{\rho \nu} .
$$

As discussed above, we can absorb $\xi^{\mu}$ in

$$
\tilde{A}_{\nu \rho \sigma}=A_{\nu \rho \sigma}+A_{\nu \rho \sigma}[\xi], \quad A_{\nu \rho \sigma}[\xi]=\frac{1}{\mathcal{R}^{2}}\left(P_{\mathrm{SD}}\right)_{\rho \sigma}^{\rho^{\prime} \sigma^{\prime}} g_{\nu \sigma^{\prime}} \xi_{\rho^{\prime}}
$$

Here $P_{\mathrm{SD}}$ is the projector on the SD antisymmetric component. Similarly, the $\mathbf{A}_{5}$ modes can absorb the radial $\kappa$ modes for the generic spheres, but should be dropped for $S_{N}^{4}$ since the corresponding fluctuations $\mathcal{A}^{\mu} \sim P^{\mu} A_{5}$ vanish.

We will see that the $A_{\mu}$ describes a U(1) gauge field and $h_{\mu \nu}$ determines the metric fluctuations, while $a_{\mu \nu}$ does not seem to play a significant role. $A_{\mu \nu \rho}$ is part of the gravitational sector. It is important to keep in mind that (apart from the $\xi^{\mu}$ and the $\kappa$ deformations) these deformation modes are "internal" degrees of freedom, whose averages $[.]_{0}$ over the local fiber vanishes. Some of these deformations are sketched in figure 1 . The only modes which change the embedding of $S^{4}$ in target space are the radial modes $\kappa$. The organization (3.17) is quite general and applies also to other covariant quantum spaces, even with Lorentzian signature. The full expansion into higher spin modes is obtained by allowing the $\mathbf{A}_{\mu}, \xi^{\mu}$ and $\kappa$ fields to take values in the universal enveloping algebra of $\mathfrak{s o}(5)$ or $I \mathfrak{s o}(4)$.

\subsection{Gauge transformation}

Consider gauge transformations

$$
Y^{a} \rightarrow Y^{a}+i\left[\Lambda, Y^{a}\right]
$$


with some gauge parameter $\Lambda \in \operatorname{End}(\mathcal{H})$. For fluctuations on a background $Y^{a}=X^{a}+\mathcal{A}^{a}$, this leads to the inhomogeneous transformation

$$
\delta \mathcal{A}^{a}=i\left[\Lambda, X^{a}\right]+i\left[\Lambda, \mathcal{A}^{a}\right]
$$

We can expand the gauge parameter in $\mathrm{SO}(5)$ generators as

$$
\Lambda=\Lambda_{0}+\frac{1}{2} \Lambda_{a b} \mathcal{M}^{a b}+\ldots
$$

where $\Lambda_{0}, \Lambda_{a b} \in \mathcal{C}_{N}\left(S^{4}\right)$. Clearly $\Lambda_{a b} \mathcal{M}^{a b}$ generates an $x$-dependent $\mathrm{SO}(5)$ transformation, and

$$
\mathbf{A}_{a}=A_{a}(x)+A_{a b c}(x) \mathcal{M}^{b c}
$$

transforms as (noncommutative) $\mathrm{SO}(5) \times \mathrm{U}(1)$-valued gauge field.

Local SO(4) rotations \& diffeomorphisms. It is interesting to work out the explicit form of these transformations in the local $4 \mathrm{D}$ parametrization (3.17). We expand

$$
\Lambda=\Lambda_{0}+v_{\mu} P^{\mu}+\frac{1}{2} \Lambda_{\mu \nu} \mathcal{M}^{\mu \nu}+\ldots
$$

and define the individual transformations

$$
\begin{aligned}
\delta_{\Lambda_{0}} & :=i\left[\Lambda_{0}, .\right] \\
\delta_{v} & :=i\left[v_{\rho} P^{\rho}, .\right], \\
\delta_{\Lambda} & :=\frac{i}{2}\left[\Lambda_{\rho \sigma} \mathcal{M}^{\rho \sigma}, .\right] .
\end{aligned}
$$

In the semi-classical limit, we can replace the commutators by Poisson brackets, and

$$
\begin{aligned}
\delta_{\Lambda_{0}} X^{\mu} & =\theta^{\mu \nu} \partial_{\nu} \Lambda_{0} \\
\delta_{v} X^{\mu} & =-v^{\mu}+\theta^{\mu \nu}\left(\partial_{\nu} v_{\rho}\right) P^{\rho} \\
\delta_{v} \phi & =i\left[v_{\rho} P^{\rho}, \phi\right] \sim-v^{\rho} \partial_{\rho} \phi+\theta^{\mu \nu}\left(\partial_{\mu} \phi\right)\left(\partial_{\nu} v_{\rho} P^{\rho}\right) \\
\delta_{\Lambda} X^{\mu} & =\frac{1}{2} i\left[\Lambda_{\rho \sigma} \mathcal{M}^{\rho \sigma}, X^{\mu}\right] \sim \frac{1}{2} \theta^{\mu \nu} \partial_{\nu} \Lambda_{\rho \sigma} \mathcal{M}^{\rho \sigma} \\
\delta_{\Lambda} \phi & =\frac{1}{2} i\left[\Lambda_{\rho \sigma} \mathcal{M}^{\rho \sigma}, \phi\right] \sim O(\theta \partial \phi \partial \Lambda)
\end{aligned}
$$

where $\phi=\phi(X)$ indicates some scalar field, and $\theta^{\mu \nu}$ is the undeformed Poisson tensor. Here we recalled that $x^{\mu}=0=\delta_{\Lambda} x^{\mu}$ at $p$. Restricted to the lowest degree in $\theta$, the $\delta_{v}$ clearly acts as a diffeomorphisms on scalar fields $\phi(x)$, and $\delta_{\Lambda}$ leads to local $\mathrm{SO}(4)$ rotations of tensors (which vanishes for scalar functions at the north pole). Applying this to the background $X+\mathcal{A}$, we can read off the transformations of the tangential and radial perturbations

$$
\begin{aligned}
& \delta \mathcal{A}^{\mu}=\theta^{\mu \nu} \partial_{\nu}\left(\Lambda_{0}+v_{\rho} P^{\rho}+\frac{1}{2} \Lambda_{\sigma \rho} \mathcal{M}^{\sigma \rho}\right)-v^{\mu}+\delta_{v} \mathcal{A}^{\mu}+\delta_{\Lambda} \mathcal{A}^{\mu}+\delta_{\Lambda_{0}} \mathcal{A}^{\mu} \\
& \delta \mathcal{A}^{5}=\delta_{v} \kappa+\delta_{\Lambda} \kappa+\delta_{\Lambda_{0}} \kappa
\end{aligned}
$$


where

$$
\begin{aligned}
\delta_{v}\left(\theta^{\mu \nu} \mathbf{A}_{\nu}\right) & =i\left[v_{\rho} P^{\rho}, \theta^{\mu \nu}\right] \mathbf{A}_{\nu}+\theta^{\mu \nu} \delta_{v} \mathbf{A}_{\nu} \\
& =v_{\rho} r^{2}\left(-g^{\rho \mu} P^{\nu}+g^{\rho \nu} P^{\mu}\right) \mathbf{A}_{\nu}+\theta^{\mu \nu} \delta_{v} \mathbf{A}_{\nu} \\
\delta_{\Lambda}\left(\theta^{\mu \nu} \mathbf{A}_{\nu}\right) & =i \frac{1}{2}\left[\Lambda_{\sigma \rho} \mathcal{M}^{\rho \sigma}, \theta^{\mu \nu}\right] \mathbf{A}_{\nu}+\theta^{\mu \nu} \delta_{\Lambda} \mathbf{A}_{\nu} \\
& =-\Lambda_{\sigma \rho}\left(g^{\rho \mu} \theta^{\sigma \nu}-g^{\rho \nu} \theta^{\sigma \mu}\right) \mathbf{A}_{\nu}+\theta^{\mu \nu} \delta_{\Lambda} \mathbf{A}_{\nu} \\
& =(\Lambda \cdot \theta \mathbf{A})^{\mu}+\theta^{\mu \nu}(\Lambda \cdot \mathbf{A})_{\nu} .
\end{aligned}
$$

Here we denote the local rotation of $\mathbf{A}_{\mu}$ by $\Lambda \in \mathfrak{s o}(4)$ with

$$
(\Lambda \cdot \mathbf{A})_{\mu}:=-\Lambda_{\mu \rho} g^{\rho \nu} \mathbf{A}_{\nu}+\ldots
$$

which extends to all the tensor legs of $\mathbf{A}_{\mu}$ in the expansion (3.17). Dropping contributions to the higher $\xi^{\mu}$ modes which we don't keep track of, we obtain the following linearized gauge transformations for the $4 \mathrm{D}$ fields

$$
\begin{aligned}
\delta \mathbf{A}_{\mu} & =\partial_{\mu}\left(\Lambda_{0}+v_{\rho} P^{\rho}+\frac{1}{2} \Lambda_{\sigma \rho} \mathcal{M}^{\sigma \rho}\right)-v^{\rho} \partial_{\rho} \mathbf{A}_{\mu}+(\Lambda \cdot \mathbf{A})_{\mu}-v_{\rho} \theta^{\rho \mu} \\
\delta \kappa & =-v^{\rho} \partial_{\rho} \kappa \\
\delta \xi^{\mu} & =-v^{\mu} .
\end{aligned}
$$

Separating $\mathbf{A}_{\mu}$ into the tensor components, this gives

$$
\begin{aligned}
\delta A_{\mu} & =\partial_{\mu} \Lambda_{0}-v^{\rho} \partial_{\rho} A_{\mu}+(\Lambda \cdot A)_{\mu} \\
\delta a_{\mu \nu} & =\left(\partial_{\mu} v_{\rho}-\partial_{\rho} v_{\mu}\right)-v^{\rho} \partial_{\rho} a_{\mu \nu}+(\Lambda \cdot a)_{\mu \nu} \\
\delta h_{\mu \nu} & =\left(\partial_{\mu} v_{\rho}+\partial_{\rho} v_{\mu}\right)-v^{\rho} \partial_{\rho} h_{\mu \nu}+(\Lambda \cdot h)_{\mu \nu} \\
\delta A_{\mu \rho \sigma} & =\frac{1}{2} \partial_{\mu} \Lambda_{\sigma \rho}(x)-v^{\rho} \partial_{\rho} A_{\mu \rho \sigma}+(\Lambda \cdot A)_{\mu \rho \sigma} .
\end{aligned}
$$

These can be understood as local $\mathrm{SO}(4)$ rotations generated by $\Lambda_{\mu \nu}(x), \mathrm{U}(1)$ gauge transformations generated by $\Lambda_{0}(x)$, and infinitesimal diffeomorphisms generated by $-v^{\rho} \partial_{\rho}$. The $A_{\mu \rho \sigma}$ transforms like a $\mathrm{SO}(4)$ gauge field. The inhomogeneous transformation of $h_{\mu \nu}$ under diffeomorphisms can be understood by anticipating that it plays the role of a linearized metric fluctuation $g_{\mu \nu}-h_{\mu \nu}$; its transformation by $v$ then gives

$$
\delta\left(g_{\mu \nu}-h_{\mu \nu}\right)=g_{\mu \nu}-h_{\mu \nu}-\left(\partial_{\mu} v_{\rho}+\partial_{\rho} v_{\mu}+v^{\rho} \partial_{\rho}\left(g_{\mu \nu}-h_{\mu \nu}\right)\right),
$$

which is the transformation of the metric tensor $g_{\mu \nu}-h_{\mu \nu}$ under an infinitesimal diffeomorphism $-v^{\rho} \partial_{\rho}$.

Higher-order gauge transformations. The gauge transformations considered in (3.24) are only the lowest in a whole tower. Consider e.g. the transformations generated by

$$
\Lambda=\Lambda_{\alpha \beta} P^{\alpha} P^{\beta}
$$


which leads to

$$
\left[X^{\mu}, \Lambda\right]=-i \Lambda_{\alpha \beta}\left(g^{\mu \alpha} P^{\beta}+g^{\mu \beta} P^{\alpha}\right)+\left[X^{\mu}, \Lambda_{\alpha \beta}\right] P^{\alpha} P^{\beta} .
$$

This allows to gauge away the symmetric $\xi^{\mu \nu}$ modes. In contrast, one cannot gauge away the $h_{\mu \nu}$ modes. From a geometric point of view, the pure gauge modes correspond to Hamiltonian vector fields on $\mathbb{C} P^{3}$, and a systematic analysis is postponed for future work.

Gauge fixing. The gauge fixing was achieved by adding the Faddeev-Popov (or BRST) gauge fixing term $-f^{2}$ to the action, such that the explicit $f^{2}$ term in (3.4) is canceled. This ensures that the propagator is well-defined. The corresponding gauge fixing condition $0=i\left[X_{a}, \mathcal{A}^{a}\right]$ is accordingly not a "hard constraint", but simply selects the physical Hilbert space or configuration space without redundancies.

Now consider the gauge fixing condition

$$
0=i\left[X_{a}, \mathcal{A}^{a}\right]=i\left[X_{5}, \mathcal{A}^{5}\right]+i\left[X_{\mu}, \theta^{\mu \nu} \mathbf{A}_{\nu}\right] .
$$

The radial contribution from $\kappa$ is

$$
i\left[X^{a}, \frac{X_{a}}{R} \kappa\right] \sim-\left\{x^{5}, \kappa\right\}=\theta^{\mu 5} \partial_{\mu} \kappa=\frac{1}{2} r \Delta^{2} P^{\mu} \partial_{\mu} \kappa,
$$

thus the gauge fixing condition is

$$
0 \sim-\frac{1}{4} \Delta^{4} g^{\nu \mu} \partial_{\mu} \mathbf{A}_{\nu}+\frac{1}{2} r \Delta^{2} P^{\mu} \partial_{\mu} \kappa+\theta^{\mu \nu} A_{\nu \mu} .
$$

Separating the components, this leads to

$$
\begin{aligned}
& 0=\partial^{\mu} A_{\mu} \\
& 0=\frac{\Delta^{2}}{2} \partial^{\mu} A_{\mu \nu}-r \partial_{\nu} \kappa \\
& 0=\theta^{\mu \nu}\left(\frac{1}{2} a_{\nu \mu}-R^{2} \partial^{\rho} \tilde{A}_{\rho \mu \nu}\right)
\end{aligned}
$$

For $a_{\mu \nu}=0$, these reduce to the Lorentz gauge condition for $A_{\mu}$ and $A_{\rho \mu \nu}$ while the second condition reduces to $\partial^{\mu} h_{\mu \nu}=\frac{2}{R} \partial_{\nu} \kappa$.

\section{Geometry: metric and vielbein}

Undeformed background. Consider some scalar field $\phi=\phi(X)$. The adjoint action of the basic matrices $\left[X^{a},.\right]$ defines a derivative operator on $\phi$,

$$
D^{a} \phi:=-i\left[X^{a}, \phi\right] \sim e^{a \mu} \partial_{\mu} \phi .
$$

where

$$
e^{a \mu}=\theta^{a \mu}, \quad e^{a}=e^{a \mu} \partial_{\mu}
$$

plays the role of a vielbein or frame. Using (2.40), we see that the tangential vielbeins

$$
e^{\alpha} \sim \theta^{\alpha \mu} \partial_{\mu}, \quad \alpha=1, \ldots, 4
$$


play the dominant role, while the transversal component $e^{5} \sim \theta^{5 \mu} \partial_{\mu}$ only contributes a small multiplicative factor $\frac{L_{R}^{2}}{R^{2}}$ to $\gamma^{\mu \nu}$ via (2.39). Recalling the discussion in section 2.3, this vielbein arises from the bundle of (selfdual) 2-tensors ${ }^{8} \theta^{\mu \nu}$, which transform in the $(1,0)$ under $\mathrm{SO}(4)$ along the internal fiber $S^{2}$. Hence $e^{\alpha \mu}$ is not a fixed frame on $S^{4}$, but it is averaged out over the fiber, $\left[e^{\mathrm{e} \nu}\right]_{0}=0$. We can now rewrite the formula for the metric $(2.35)$ on $\mathcal{S}_{\Lambda}^{4}$ in a more suggestive way as follows

$$
\bar{\gamma}^{\mu \nu}=g_{a b} \theta^{a \mu} \theta^{b \nu}=g_{a b} e^{a \mu} e^{b \nu}
$$

This defines a fixed, well-defined metric on $S^{4}$ which is constant along the fiber $S^{2}$,

$$
\left[\bar{\gamma}^{\mu \nu}\right]_{0}=\bar{\gamma}^{\mu \nu}=\frac{\Delta^{4}}{4} g^{\mu \nu} .
$$

This is the key property which allows to reconcile covariance with noncommutativity. For generic $\mathcal{S}_{\Lambda}^{4}$ with large $L_{R}$, the $\bar{\gamma}^{\mu \nu}$ is replaced by the 5-dimensional $\bar{\gamma}^{a b}$ as in (2.37).

Now consider general fields $\phi \in \operatorname{End}(\mathcal{H})$, decomposed into a tower of higher spin (tensor) fields on $S^{4}$ as in (2.26). The adjoint action $\left[X^{a},.\right]$ still defines a derivative operator $\phi$, which however contains non-derivative terms which arise from commutators of the $X^{\alpha}$ with the $P^{\mu}$ generators in the expansion of $\phi$. E.g. for $\phi=\phi+\phi_{\mu} P^{\mu}+\phi_{\mu \nu} \mathcal{M}^{\mu \nu}$, we have

$$
D^{\alpha} \phi=-i\left[X^{\alpha}, \phi+\phi_{\mu} P^{\mu}+\phi_{\mu \nu} \mathcal{M}^{\mu \nu}\right] \sim e^{\alpha \rho} \partial_{\rho} \phi-\phi^{\alpha} .
$$

This phenomenon will play a crucial role below. Nevertheless, the metric in the kinetic term for arbitrary fields is always obtained from the leading derivative contributions

$$
-\left[X^{a}, \phi\right]\left[X_{a}, \phi\right]=\bar{\gamma}^{\mu \nu} \partial_{\mu} \phi \partial_{\nu} \phi+\ldots
$$

Deformed background. Now we include the fluctuations $Y^{a}=X^{a}+\mathcal{A}^{a}$. Since the kinetic term for (bosonic) fields always arise from contracted commutators

$$
-\left[Y^{a}, \phi\right]\left[Y_{a}, \phi\right] \sim D^{a} \phi D_{a} \phi=\gamma^{\mu \nu} \partial_{\mu} \phi \partial_{\nu} \phi+\ldots, \quad D^{a}:=-i\left[Y^{a}, .\right]
$$

we can read off the effective metric in the perturbed matrix model (up to a possible conformal factor, see below)

$$
\gamma^{\mu \nu} \sim-\left[Y^{a}, X^{\mu}\right]\left[Y_{a}, X^{\nu}\right]=D^{a} x^{\mu} D_{a} x^{\nu} .
$$

and similarly for the 5D case with $\gamma^{a b}$. This can be expressed in terms of an (over-complete) frame

$$
e^{a}[\mathcal{A}]=e^{a \mu}[\mathcal{A}] \partial_{\mu}=D^{a}
$$

cf. $[8,39]$. Again the tangential contributions $e^{\alpha}[\mathcal{A}], \alpha=1, \ldots, 4$ will provide the leading contribution. Recall the explicit form of tangential fluctuations $\mathcal{A}^{\alpha}=\frac{x^{\alpha}}{R} \kappa+\theta^{\alpha \mu} \mathbf{A}_{\mu}$ with

$$
\mathbf{A}_{\mu}=A_{\mu}+A_{\mu \nu} P^{\nu}+\tilde{A}_{\mu \rho \sigma} \mathcal{M}^{\rho \sigma},
$$

\footnotetext{
${ }^{8}$ Note that $\mathcal{J}_{\alpha}^{\mu}:=e_{\alpha}^{\mu}$ can be interpreted as (rescaled) almost-complex structure, since $\mathcal{J}^{2} \sim-\mathbb{1}$. Again this does not live on $S^{4}$ but on the bundle $\mathcal{O}(\Lambda)$.
} 
Observe first

$$
-i\left[\mathbf{A}_{\mu}, \phi\right] \sim\left(A_{\mu \beta} g^{\beta \nu}+\theta^{\beta \nu} \partial_{\beta} \mathbf{A}_{\mu}\right) \partial_{\nu} \phi
$$

where the non-derivative $A_{\mu \nu}$ term arises from the explicit $P$ modes in $\mathbf{A}_{\mu}$, similar as in (4.6). Using these expressions and dropping as usual $\left[\mathcal{M}^{\alpha \beta}, \phi\right]=O\left(\frac{x}{R} \partial \phi\right)$ in the local frame, we obtain ${ }^{9}$

$$
\begin{aligned}
D^{\alpha} \phi & \sim-i\left[Y^{\alpha}, \phi\right]=-i\left[X^{\alpha}\left(1+\frac{\kappa}{R}\right)+\mathcal{A}^{\alpha}, \phi\right] \\
& \sim\left(\theta^{\alpha \nu}\left(1+\frac{\kappa}{R}\right)+\theta^{\alpha \mu} A_{\mu \beta} g^{\beta \nu}+\theta^{\alpha \mu} \theta^{\beta \nu}\left(\partial_{\beta} A_{\mu}+\partial_{\beta} \tilde{A}_{\mu \rho \sigma} \mathcal{M}^{\rho \sigma}\right)\right) \partial_{\nu} \phi-\phi^{\alpha} \\
& =e^{\alpha \nu}[\mathcal{A}] \partial_{\nu} \phi-\phi^{\alpha} .
\end{aligned}
$$

Hence the tangential vielbein $e^{\alpha}[\mathcal{A}]=e^{\alpha \nu}[\mathcal{A}] \partial_{\nu}$ is

$$
\begin{aligned}
e^{\alpha \nu}[\mathcal{A}] & \sim-i\left[Y^{\alpha}, X^{\nu}\right] \\
& \sim \theta^{\alpha \mu}\left(\delta_{\mu}^{\nu}\left(1+\frac{\kappa}{R}\right)+A_{\mu \beta} g^{\beta \nu}+\theta^{\beta \nu}\left(\partial_{\beta} A_{\mu}+\partial_{\beta} \tilde{A}_{\mu \rho \sigma} \mathcal{M}^{\rho \sigma}\right)\right) .
\end{aligned}
$$

The transversal vielbein

$$
e^{5 \nu}[\mathcal{A}] \sim-i\left[Y^{5}, X^{\nu}\right] \sim \theta^{5 \nu}+\theta^{\mu \nu} \partial_{\mu} \kappa
$$

does not contribute to the linearized metric perturbations and can be dropped. Hence the tangential $e^{\alpha}, \alpha=1, \ldots, 4$ play the role of the effective vielbein. Using these results, the metric on a deformed $\mathcal{S}_{\Lambda}^{4}$ background including linearized perturbations is

$$
\gamma^{\mu \nu} \sim-\left[Y^{\alpha}, X^{\mu}\right]\left[Y_{\alpha}, X^{\nu}\right]=g_{\alpha \beta} e^{\alpha \mu} e^{\beta \nu}=\bar{\gamma}^{\mu \nu}+\delta \gamma^{\mu \nu}
$$

where the linearized metric fluctuations are given by

$$
\delta \gamma^{\mu \nu}=\frac{\Delta^{4}}{4}\left(h^{\mu \nu}+\frac{2 \kappa}{R} g^{\mu \nu}\right)+\frac{\Delta^{4}}{4}\left(g^{\mu \nu^{\prime}} \theta^{\beta \nu}\left(\partial_{\beta} A_{\nu^{\prime}}+\partial_{\beta} \tilde{A}_{\nu^{\prime} \rho \sigma} \mathcal{M}^{\rho \sigma}\right)+(\mu \leftrightarrow \nu)\right)
$$

using (2.35), always raising and lowering indices with $g^{\mu \nu}$. Note that the anti-symmetric contributions $a_{\mu \nu}$ drop out. After averaging over the fiber $S^{2}$ using (C.1), the contribution from the $\mathrm{U}(1)$ gauge field $A_{\nu}$ also drops out since $\left[\theta^{\beta \nu}\right]_{0}=0$, and we obtain

$$
\left[\delta \gamma^{\mu \nu}\right]_{0}=\frac{\Delta^{4}}{4}\left(h^{\mu \nu}+k^{\mu \nu}+\frac{2 \kappa}{R} g^{\mu \nu}\right)=: \frac{\Delta^{4}}{4} \tilde{h}^{\mu \nu} .
$$

The contribution from the rank 3 tensor $\tilde{A}_{\mu \rho \nu}$ is

$$
k_{\mu \nu}:=g^{\mu \nu^{\prime}}\left[\theta^{\beta \nu} \mathcal{M}^{\rho \sigma}\right]_{0} \partial_{\beta} \tilde{A}_{\nu^{\prime} \rho \sigma}+(\mu \leftrightarrow \nu)=\frac{4 R^{2}}{3}\left(\partial^{\rho} \tilde{A}_{\mu \rho \nu}+\partial^{\rho} \tilde{A}_{\nu \rho \mu}\right)
$$

using $\frac{\Delta^{4}}{4}=r^{2} R^{2}$ as well as the self-duality of $\tilde{A}_{\mu \rho \sigma}$ in the last indices. Note that $k_{\mu \nu}$ transforms as

$$
k_{\mu \nu} \rightarrow k_{\mu \nu}+\partial_{\mu} v_{\nu}+\partial_{\nu} v_{\mu}, \quad v_{\nu}=\frac{4 R^{2}}{3} \partial^{\rho} \Lambda_{\nu \rho}
$$

under local SO(4) gauge transformations, and the gauge condition (3.38) for $\tilde{A}_{\mu \rho \nu}$ implies

$$
\partial^{\mu} k_{\mu \nu}=0 \quad \text { if } \quad a_{\mu \nu}=0 .
$$

\footnotetext{
${ }^{9}$ We include also the term $\phi^{\mu} P_{\mu}$ in the expansion of $\phi \in E n d(\mathcal{H})$, which is needed for the field strength where $\phi \rightarrow \mathcal{A}$. The term $\partial_{\beta} A_{\mu \rho} P^{\rho}$ is dropped since it would drop out upon averaging over $S^{2}$ in (4.18).
} 


\subsection{Thick spheres, extra dimensions and dimensional reduction}

Since the underlying space $\mathcal{O}(\Lambda)$ is higher-dimensional, there are excitation modes in extra dimensions. Most of them give rise to higher spin modes, as discussed before. However for the generalized spheres $\mathcal{S}_{\Lambda}^{4}$, there are also extra scalar modes, corresponding to the $\mathrm{SO}(5)$ Casimir $\mathcal{R}^{2}$. They arise from the $\mathfrak{s o}(6)$ mode decomposition (2.27) reduced to $\mathfrak{s o}(5)$.

Now the stabilization of the spheres becomes important. As shown in [36], there is a one-loop effective potential $V\left(\mathcal{R}^{2}\right)$, which for $\mathcal{S}_{N}^{4}$ - assuming some bare mass $\mu^{2}$ - acquires a non-trivial minimum $R$. Here we will actually need "thick" spheres $\mathcal{S}_{\Lambda}^{4}$, which will clearly also have some radial potential. Due to the thickness, the long-wavelength modes in the radial directions will be significantly suppressed by this $\mathcal{V}(R)$, while modes localized at $R$ will have large kinetic energy. This suggests that there should be a large mass gap in the radial direction.

There is another attractive mechanism to get rid of the extra internal modes along the lines of [31, 40,41], by giving suitable VEV's to the transversal matrices or scalar fields. This leads to fuzzy extra dimensions with a large mass gap, ${ }^{10}$ and at the same time lead to an interesting low-energy gauge theory. For example, the 6 transversal matrices in the IKKT model could be identified with the generators of squashed $\mathbb{C} P^{2}[41,42]$, since $\mathcal{S}_{\Lambda}^{4}$ is a $\mathbb{C} P^{2}$-bundle over $\mathcal{S}_{N}^{4}$, see appendix A. This will be studied elsewhere. Here we will simply proceed in the framework of the dimensionally reduced 4-dimensional theory, and elaborate the resulting 4-dimensional gravity.

\subsection{Effective metric and scalar fields}

To properly identify the effective metric, consider scalar fields propagating on the deformed $\mathcal{S}_{\Lambda}^{4}$ background in more detail. The kinetic term for a (transversal) scalar field is

$$
S[\phi]=-\frac{2}{g^{2}} \operatorname{Tr}\left[Y^{a}, \phi\right]\left[Y_{a}, \phi\right] \sim \frac{\operatorname{dim} \mathcal{H}}{\operatorname{Vol}\left(\mathcal{M}^{4}\right)} \frac{2}{g^{2}} \int_{\mathcal{M}} d^{4} x \gamma^{\mu \nu} \partial_{\mu} \phi \partial_{\nu} \phi
$$

To be specific, we use the Riemannian measure in target space. We can cast this into a covariant-looking form (cf. [8])

$$
\begin{aligned}
S[\phi] & \sim \frac{\operatorname{dim} \mathcal{H}}{\operatorname{Vol}\left(\mathcal{M}^{4}\right)} \frac{\Delta^{4}}{2 g^{2}} \int_{\mathcal{M}} d^{4} x \sqrt{\left|G_{\mu \nu}\right|} G^{\mu \nu} \partial_{\mu} \phi \partial_{\nu} \phi \\
& =\frac{1}{2} \int_{\mathcal{M}} d^{4} x \sqrt{\left|G_{\mu \nu}\right|} G^{\mu \nu} \partial_{\mu} \varphi \partial_{\nu} \varphi
\end{aligned}
$$

in terms of the effective metric

$$
G^{\mu \nu}=\alpha \frac{4}{\Delta^{4}} \gamma^{\mu \nu}, \quad \alpha=\sqrt{\frac{\Delta^{4}}{4}\left|\gamma_{\mu \nu}^{-1}\right|}=1-\frac{1}{2} \tilde{h}+\ldots
$$

which is a dimensionless Weyl rescaling of $\gamma^{\mu \nu}$, and a field $\varphi$ which has dimension mass via

$$
\phi=\frac{\Delta^{2} g_{\mathrm{YM}}}{2 \sqrt{2}} \varphi .
$$

\footnotetext{
${ }^{10}$ The gravitational modes are protected from acquiring a mass by gauge invariance.
} 
(which has the usual dimension of mass). Here $g_{\mathrm{YM}}$ is defined in (4.36), and the corresponding linearized metric fluctuation is obtained from (4.18)

$$
G^{\mu \nu}=g^{\mu \nu}+H^{\mu \nu}, \quad H^{\mu \nu}=\tilde{h}^{\mu \nu}-\frac{1}{2} g^{\mu \nu} \tilde{h} .
$$

where $\tilde{h}_{\mu \nu}=h_{\mu \nu}+k_{\mu \nu}$. Then the Lorentz-gauge condition $\partial_{\mu} \tilde{h}^{\mu \nu}=0$ translates into the de Donder gauge for $H_{\mu \nu}$,

$$
\partial^{\mu} H_{\mu \nu}-\frac{1}{2} \partial_{\nu} H=0
$$

We consider $H_{\mu \nu}$ as a tensor field here, rising and lowering indices with $g_{\mu \nu}$. Then the linearized coupling of $\tilde{h}_{\mu \nu}$ to matter is given by

$$
\delta_{h} S[\phi]=\frac{1}{2} \int_{\mathcal{M}} d^{4} x H^{\mu \nu} T_{\mu \nu}[\varphi]=\frac{1}{2} \int_{\mathcal{M}} d^{4} x \tilde{h}^{\mu \nu}\left(T_{\mu \nu}[\varphi]-\frac{1}{2} g_{\mu \nu} T\right)
$$

where

$$
T_{\mu \nu}[\varphi]=\partial_{\mu} \varphi \partial_{\nu} \varphi-\frac{1}{2} g_{\mu \nu}\left(g^{\rho \sigma} \partial_{\rho} \varphi \partial_{\sigma} \varphi\right), \quad T=-g^{\mu \nu} \partial_{\mu} \varphi \partial_{\nu} \varphi
$$

is the energy-momentum tensor of $\varphi$, which satisfies $\partial^{\mu} T_{\mu \nu}=0$.

\subsection{Flux and field strength}

Now consider the perturbation of the "flux" $\left[Y^{\mu}, Y^{\nu}\right] \sim i \Theta_{(Y)}^{\mu \nu}$ given by

$$
\begin{aligned}
\Theta^{\mu \nu} & =\theta^{\mu \nu}+\mathcal{F}^{\mu \nu}=\tilde{\theta}^{\mu \nu}[A]+\theta^{\mu \mu^{\prime}} \theta^{\nu \nu^{\prime}} F_{\mu^{\prime} \nu^{\prime}}[\mathbf{A}] \\
\tilde{\theta}^{\mu \nu} & :=\theta^{\mu \nu}+\theta^{\mu \mu^{\prime}} A_{\mu^{\prime} \nu^{\prime}} g^{\nu^{\prime} \nu}-\theta^{\nu \nu^{\prime}} A_{\nu^{\prime} \mu^{\prime}} g^{\mu^{\prime} \mu} \\
& =\left(\delta_{\mu^{\prime}}^{\mu}+A_{\mu^{\prime} \rho} g^{\mu \rho}\right) \theta^{\mu^{\prime} \nu^{\prime}}\left(\delta_{\nu^{\prime}}^{\nu}+A_{\nu^{\prime} \rho} g^{\rho \nu}\right)
\end{aligned}
$$

to linearized order. Since the $A$ terms enter through one factor of $\theta^{\mu \mu^{\prime}}$, they are naturally viewed as geometric deformation of the background $\theta^{\mu \nu} \rightarrow \tilde{\theta}^{\mu \nu}$, which plays the role of the Poisson tensor in the deformed $y^{\mu}$ coordinates. In contrast, the field strength

$$
\begin{aligned}
F_{\mu \nu}[\mathbf{A}] & =\partial_{\mu} \mathbf{A}_{\nu}-\partial_{\nu} \mathbf{A}_{\mu}-i\left[A_{\mu}, A_{\nu}\right] \\
& =F_{\mu \nu}+R_{\mu \nu}+T_{\mu \nu}
\end{aligned}
$$

enters via 2 factors of $\theta$, and decomposes into the $\mathrm{U}(1) \times I \mathfrak{s o}(4)$-valued components

$$
\begin{aligned}
F_{\mu \nu} & =\partial_{\mu} A_{\nu}-\partial_{\nu} A_{\mu} & & \\
R_{\mu \nu} & =\partial_{\mu} \omega_{\nu}-\partial_{\nu} \omega_{\mu}-i\left[\omega_{\mu}, \omega_{\nu}\right], & & \omega_{\mu}=A_{\mu \alpha \beta} \mathcal{M}^{\alpha \beta} \\
T_{\mu \nu} & =\partial_{\mu} \alpha_{\nu}-\partial_{\nu} \alpha_{\mu}-i\left(\left[\omega_{\mu}, \alpha_{\nu}\right]-\left[\omega_{\nu}, \alpha_{\mu}\right]\right), & & \alpha_{\mu}=A_{\mu \alpha} P^{\alpha} .
\end{aligned}
$$

Clearly $F_{\mu \nu}$ is the U(1) field strength of $A_{\mu}$, and $R_{\mu \nu}$ is the curvature of $A_{\mu \alpha \beta} \mathcal{M}^{\alpha \beta}$ viewed as $\mathfrak{s o}(4)$ connection. Furthermore, $T_{\mu \nu}$ can be related to the linearized spin connection. 
Stack of branes and nonabelian gauge theory. As usual, $\mathrm{SU}(k)$ gauge fields can be obtained by considering a stack of coincident branes, expanding the fluctuations as $A_{\mu}=A_{\mu} \mathbb{1}+A_{\mu, a} \lambda^{a}$ in terms of $\mathfrak{s u}(k)$ generators $\lambda^{a}$. For the generic $\mathcal{S}_{\Lambda}^{4}$ spheres there is no need to do this by hand, since they can be interpreted as bundles over $\mathcal{S}_{N}^{4}$ with fibers being fuzzy coadjoint orbits of SU(3) (see appendix A for more details, and [43] for an explicit example in a simplified setting). This means that some non-trivial gauge theory will arise automatically, whose structure is similar to the squashed brane configurations in [41, 42], which in turn are quite close to the standard model. It is very remarkable that the $\mathcal{S}_{\Lambda}^{4}$ spheres seem to provide the right ingredients for both gravity and particle physics.

For such nonabelian gauge fields arising on $\mathcal{S}_{\Lambda}^{4}$, the $\mathfrak{u}(k)$-valued fluctuations should be expanded in terms of ${ }^{11}$

$$
\mathcal{A}^{\alpha}=\tilde{e}^{\alpha \nu} A_{\nu}
$$

rather than $\mathcal{A}^{\alpha}=\theta^{\alpha \nu} A_{\nu}$. Here

$$
\tilde{e}^{\alpha \mu}[A]=\sqrt{\alpha} \frac{2}{\Delta^{2}} e^{\alpha \mu}[A]
$$

is the conformally rescaled dimensionless vielbein corresponding to the effective metric $G^{\mu \nu}$ (4.24). Then the correct coupling to the metric is recovered (cf. [8]),

$$
S[F]=\frac{1}{g^{2}} \operatorname{tr}\left[Y^{\mu}, Y^{\nu}\right]\left[Y_{\mu}, Y_{\nu}\right] \sim \frac{1}{4 g_{\mathrm{YM}}^{2}} \int_{\mathcal{M}} d^{4} x \sqrt{|G|} G^{\mu \nu} G^{\mu^{\prime} \nu^{\prime}} F_{\mu \mu^{\prime}} F_{\nu \nu^{\prime}} .
$$

where the Yang-Mills coupling constant is defined as ${ }^{12}$

$$
\frac{1}{4 g_{\mathrm{YM}}^{2}}=\frac{\operatorname{dim} \mathcal{H}}{\operatorname{Vol}\left(\mathcal{M}^{4}\right)} \frac{\Delta^{8}}{16 g^{2}}
$$

Noting that the conformal factor drops out in the Yang-Mills action, the linearized coupling to the metric perturbation $h_{\mu \nu}$ gives

$$
\delta_{h} S[F]=\frac{1}{2} \int_{\mathcal{M}} d^{4} x h^{\mu \nu} T_{\mu \nu}[F]
$$

where

$$
T_{\mu \nu}[F]=\frac{1}{g_{\mathrm{YM}}^{2}}\left(F_{\mu \mu^{\prime}} F_{\nu \nu^{\prime}} G^{\mu^{\prime} \nu^{\prime}}-\frac{1}{4} G^{\mu \nu}(F F)\right)
$$

is the energy momentum tensor of the gauge fields.

\section{Gravity}

\subsection{Classical action and equations of motion}

In order to derive the equations of motion for the gravitational $A_{\mu \nu}, A_{\mu \rho \sigma}$ and $\kappa$ modes, we evaluate the semi-classical action up to quadratic order. The quadratic fluctuations are

\footnotetext{
${ }^{11}$ This expansion should be applied also in (3.16) to go beyond the present linearized approximation.

${ }^{12}$ Note that the matrix model coupling $g^{2}$ has dimension $L^{4}$. For nonabelian gauge fields, an extra factor may arise from the number of branes.
} 
governed by the "vector" (matrix) Laplacian (3.6)

$$
\left(D^{2} \mathcal{A}\right)_{a}:=\left(\square+\frac{1}{2} \mu^{2}-M_{r s}^{(\mathcal{A})}\left[\Theta^{r s}, .\right]\right)_{b}^{a} \mathcal{A}_{b}
$$

Now consider the ansatz ${ }^{13}(3.17)$

$$
\mathcal{A}_{\text {grav }}^{\mu}(x)=\frac{x^{\mu}}{R} \kappa+\theta^{\mu \nu}\left(A_{\nu \sigma}(x) P^{\sigma}+\tilde{A}_{\nu \sigma \rho}(x) \mathcal{M}^{\sigma \rho}\right), \quad \mathcal{A}^{5}=\kappa
$$

dropping the $\mathrm{U}(1)$ component for now. We can evaluate $D^{2} \mathcal{A}$ in the semi-classical limit using the basic rules

$$
\begin{aligned}
{[f(x), g(x)] } & \sim i \theta^{\mu \nu} \partial_{\mu} f \partial_{\nu} g \\
{\left[\theta^{\mu \nu}, g(x)\right] } & \left.\sim O(x \partial g)\right|_{p}=0 \\
{\left[P_{\mu}, g(x)\right] } & \sim i \partial_{\mu} g
\end{aligned}
$$

which are valid in the local ON frame at $p$. After some algebra (see appendix E) and

$$
\left(\theta^{\mu \rho} \theta^{\sigma \nu}-\theta^{\mu \sigma} \theta^{\rho \nu}\right) \tilde{A}_{\nu \sigma \rho}=2 r^{2} \theta^{\mu \nu} \mathcal{M}^{\sigma \rho} \tilde{A}_{\rho \sigma \nu}
$$

using the antisymmetry of $A_{\nu \sigma \rho}$ in the last two indices, we obtain with (3.17)

$$
\begin{aligned}
D^{2} \mathcal{A}_{\text {grav }}^{\mu}= & \theta^{\mu \nu} P^{\sigma}\left(\square+8 r^{2}+\frac{1}{2} \mu^{2}\right) A_{\nu \sigma}+2 P^{\mu} \mathcal{M}^{\sigma \nu} A_{\nu \sigma}+4 \gamma^{\mu \rho} g^{\nu \sigma} \tilde{A}_{\nu \sigma \rho} \\
& +\theta^{\mu \nu} \mathcal{M}^{\sigma \rho}\left(2 r^{2} \partial_{\sigma} A_{\nu \rho}+\left(\square+8 r^{2}+\frac{1}{2} \mu^{2}\right) \tilde{A}_{\nu \sigma \rho}+4 r^{2} \tilde{A}_{\rho \sigma \nu}\right) \\
D^{2} \mathcal{A}_{\text {grav }}^{5}= & \left(\square+4 r^{2}+\frac{1}{2} \mu^{2}\right) \kappa .
\end{aligned}
$$

Now

$$
4 \gamma^{\mu \rho} g^{\nu \sigma} \tilde{A}_{\nu \sigma \rho}=-6 r^{2} \theta^{\mu \nu} \mathcal{M}^{\sigma \rho} P_{0} \tilde{A}_{\nu \sigma \rho}
$$

where $A_{\nu \sigma \rho}[\xi]=P_{0} \tilde{A}_{\nu \sigma \rho}$ is the trace contribution of $\tilde{A}_{\nu \sigma \rho}$ defined in (3.19). To evaluate $\left[\mathcal{A}_{\text {grav }} D^{2} \mathcal{A}_{\text {grav }}\right]_{0}$, we need the following normalizations which follow from (2.39) and (C.1)

$$
\begin{aligned}
{\left[P^{\sigma} \theta^{\mu \nu} g_{\mu \mu^{\prime}} \theta^{\mu^{\prime} \nu^{\prime}} P^{\sigma^{\prime}}\right]_{0} } & =L_{R}^{2} g^{\nu \nu^{\prime}} g^{\sigma \sigma^{\prime}} \\
{\left[\mathcal{M}^{\sigma \rho} \theta^{\mu \nu} g_{\mu \mu^{\prime}} \theta^{\mu^{\prime} \nu^{\prime}} \mathcal{M}^{\sigma^{\prime} \rho^{\prime}}\right]_{0} } & =\frac{R^{4}}{3} g^{\nu \nu^{\prime}}\left(g^{\sigma \sigma^{\prime}} g^{\rho \rho^{\prime}}-g^{\sigma \rho^{\prime}} g^{\rho \sigma^{\prime}}+\epsilon^{\sigma \rho \sigma^{\prime} \rho^{\prime}}\right)
\end{aligned}
$$

using $\frac{\Delta^{4}}{4}=r^{2} R^{2}$. All other mixed terms such as $\left[P^{\sigma} \theta^{\mu \nu} g_{\mu \mu^{\prime}} \theta^{\mu^{\prime} \nu^{\prime}} \mathcal{M}^{\sigma^{\prime} \rho^{\prime}}\right]_{0}$ vanish. Thus

$$
\begin{aligned}
{\left[P^{\sigma} A_{\nu \sigma} \theta^{\mu \nu} \theta^{\mu^{\prime} \nu^{\prime}} A_{\nu^{\prime} \sigma^{\prime}}^{\prime} P^{\sigma^{\prime}} g_{\mu \mu^{\prime}}\right]_{S^{2}} } & =L_{R}^{2} A^{\nu \sigma} A_{\nu \sigma}^{\prime} \\
{\left[\mathcal{M}^{\sigma \rho} A_{\nu \sigma \rho} \theta^{\mu \nu} \theta^{\mu^{\prime} \nu^{\prime}} A_{\nu^{\prime} \sigma^{\prime} \rho^{\prime}}^{\prime} \mathcal{M}^{\sigma^{\prime} \rho^{\prime}} g_{\mu \mu^{\prime}}\right]_{S^{2}} } & =\frac{4 R^{4}}{3} A^{\nu \sigma \rho} A_{\nu \sigma \rho}^{\prime}
\end{aligned}
$$

\footnotetext{
${ }^{13}$ The $x^{\mu} \frac{\kappa}{R}$ contribution is subleading here and dropped.
} 
provided either $A_{\nu \sigma \rho}$ or $A_{\nu \sigma \rho}^{\prime}$ is $\mathrm{SD}$ in the last 2 indices. Therefore the semi-classical action (3.4) quadratic in the $A_{\mu \nu}, \tilde{A}_{\nu \sigma \rho}$ and $\kappa$ fields is

$$
\begin{aligned}
S_{2}[\mathcal{A}]= & \frac{1}{g^{2}} \operatorname{Tr} \mathcal{A}_{a}\left(\left(\square+\frac{1}{2} \mu^{2}\right) \delta_{b}^{a}+2 i\left[\Theta^{a b}, .\right]\right) \mathcal{A}_{b} \\
\sim & \frac{4}{g_{\mathrm{YM}}^{2} \Delta^{8}} \int_{\mathcal{M}} d^{4} x\left(L_{R}^{2} A^{\mu \nu}\left(\square+8 r^{2}+\frac{1}{2} \mu^{2}\right) A_{\mu \nu}+\kappa\left(\square+4 r^{2}+\frac{1}{2} \mu^{2}\right) \kappa\right. \\
& \quad+\frac{4 R^{4}}{3} \tilde{A}_{\nu \sigma \rho}\left(\square+8 r^{2}+r^{2} P_{\text {hor }}-6 r^{2} P_{0}+\frac{1}{2} \mu^{2}\right) \tilde{A}^{\nu \sigma \rho}+\frac{8 \alpha R^{4}}{3} r^{2} \tilde{A}_{\nu \sigma \rho} \partial_{\sigma} A_{\nu \rho}
\end{aligned}
$$

To accommodate one-loop effects (see section 6), we introduced a factor $\alpha$ in the mixed term, which is

$$
\alpha=1
$$

for the present semi-classical action. Given in addition a coupling to matter of the form ${ }^{14}$

$$
\delta S[\phi]=\frac{1}{2} \int_{\mathcal{M}} d^{4} x\left(h^{\mu \nu}+k^{\mu \nu}+\frac{2 \kappa}{R} g^{\mu \nu}\right) T_{\mu \nu}
$$

(cf. (4.37), (4.28)) we obtain the equations of motion

$$
\begin{aligned}
L_{R}^{2}\left(\square+8 r^{2}+\frac{1}{2} \mu^{2}\right) A_{\mu \nu} & =-\frac{g_{\mathrm{YM}}^{2} \Delta^{8}}{16} T_{\mu \nu}+\frac{\alpha R^{2} \Delta^{4}}{3} \partial^{\sigma} \tilde{A}_{\mu \sigma \nu} \\
8 R^{2}\left(\square+8 r^{2}+r^{2} P_{\mathrm{hor}}-6 r^{2} P_{0}+\frac{\mu^{2}}{2}\right) A_{\nu \sigma \rho} & =\left(P_{\mathrm{SD}}\right)_{\sigma \rho}^{\sigma^{\prime} \rho^{\prime}}\left(-2 \alpha \Delta^{4} \partial_{\sigma^{\prime}} A_{\nu \rho^{\prime}}+g_{\mathrm{YM}}^{2} \Delta^{8} \partial_{\sigma^{\prime}} T_{\nu \rho^{\prime}}\right) \\
2\left(\square+4 r^{2}+\frac{1}{2} \mu^{2}\right) \kappa & =-\frac{g_{\mathrm{YM}}^{2} \Delta^{8}}{4 R} T .
\end{aligned}
$$

Here $P_{\mathrm{SD}}^{0}=\frac{1}{4}(\delta \delta-\delta \delta+\epsilon)$ is the projector on the SD antisymmetric component, $P_{0}$ is the projector on the trace contributions of $\tilde{A}_{\mu \rho \sigma}$, and $P_{\text {hor }}$ is the operator exchanging horizontal indices in the mixed hook diagram (corresponding to $\tilde{A}_{\mu \rho \sigma}$ ) defined in appendix D. This has eigenvalue $P_{\text {hor }}=-1$ on the totally antisymmetric diagrams, and $P_{\text {hor }}=\frac{1}{2}$ on the mixed (hook) Young diagrams. In any case, the contribution of $P_{\text {hor }}$ and $P_{0}$ in the kinetic operator is negligible compared with $\square$, and we neglect it for simplicity, and we replace the equation for $\tilde{A}_{\nu \sigma \rho}$ by

$$
R^{2}\left(\square+8 r^{2}+\frac{\mu^{2}}{2}\right) \tilde{A}_{\nu \sigma \rho}=\left(P_{\mathrm{SD}}\right)_{\sigma \rho}^{\sigma^{\prime} \rho^{\prime}}\left(-\frac{\alpha \Delta^{4}}{4} \partial_{\sigma^{\prime}} A_{\nu \rho^{\prime}}+\frac{g_{\mathrm{YM}}^{2} \Delta^{8}}{8} \partial_{\sigma^{\prime}} T_{\nu \rho^{\prime}}\right)
$$

This implies

$$
R^{2}\left(\square+8 r^{2}+\frac{\mu^{2}}{2}\right) \partial^{\sigma} \tilde{A}_{\nu \sigma \rho}=\frac{\alpha \Delta^{4}}{16}\left(-\partial^{\sigma} \partial_{\sigma} A_{\nu \rho}+\partial_{\rho} \partial^{\sigma} A_{\nu \sigma}\right)+\frac{g_{\mathrm{YM}}^{2} \Delta^{8}}{32} \partial^{\sigma} \partial_{\sigma} T_{\nu \rho}
$$

\footnotetext{
${ }^{14}$ For scalar fields, the coupling of the trace component $h$ was found to be modified in (4.28). This is somewhat puzzling; one possible resolution might be that the rescaling (4.25) should be done using the $x$-dependent uncertainty scale $\Delta_{x}^{2}$. This should be addressed in more detail elsewhere.
} 
Now assume that the antisymmetric component of $A_{\mu \nu}$ vanishes, $a_{\mu \nu}=0$. Then the gaugefixing condition (3.38) implies $\partial^{\sigma} A_{\nu \sigma}=\frac{1}{R} \partial_{\nu} \kappa$, so that $\partial^{\sigma} \tilde{A}_{\nu \sigma \rho}$ is symmetric in $\nu, \rho$. Then the eom (5.12) for $A_{\mu \nu}$ is indeed consistent with

$$
a_{\mu \nu}=0, \quad A_{\mu \nu}=\frac{1}{2} h_{\mu \nu}
$$

for symmetric and conserved $T_{\mu \nu}$. Putting all this together and recalling $\square \sim-\frac{\Delta^{4}}{4} \square_{g}$, we obtain the following equations for the metric contributions $k_{\mu \nu}(4.19)$ and $h_{\mu \nu}$ and $\kappa$

$$
\begin{aligned}
\left(\square_{g}-4 m^{2}\right) k_{\mu \nu} & =-\frac{g_{\mathrm{YM}}^{2} \Delta^{4}}{3} \square_{g} T_{\mu \nu}+\frac{\alpha}{3} \square_{g} h_{\mu \nu}-\frac{1}{3 R} \partial_{\mu} \partial_{\nu} \kappa \\
\left(\square_{g}-4 m^{2}\right) h_{\mu \nu} & =\frac{g_{\mathrm{YM}}^{2} \Delta^{4}}{2 L_{R}^{2}} T_{\mu \nu}-\frac{\alpha}{L_{R}^{2}} k_{\mu \nu} \\
\left(\square_{g}-4 m^{2}\right) \kappa & =\frac{g_{\mathrm{YM}}^{2} \Delta^{4}}{2 R} T .
\end{aligned}
$$

Here we define the mass

$$
m^{2}=\frac{8 r^{2}+\mu^{2} / 2}{\Delta^{4}} \stackrel{\mu^{2} \rightarrow 0}{=} \frac{1}{4 R^{2}}
$$

which is of the order of the background curvature, and can hence be dropped for simplicity. Combining the first and the second then gives

$$
\square_{g} k_{\mu \nu}=\frac{g_{\mathrm{YM}}^{2} \Delta^{4}}{3}\left(-\square_{g} T_{\mu \nu}+\frac{\alpha}{2 L_{R}^{2}} T_{\mu \nu}\right)-\frac{\alpha^{2}}{3 L_{R}^{2}} k_{\mu \nu}-\frac{1}{3 R} \partial_{\mu} \partial_{\nu} \kappa
$$

We expect from (5.16) that $\frac{1}{R} \partial_{\mu} \partial_{\nu} \kappa=O\left(\frac{g_{\mathrm{YM}}^{2} \Delta^{4}}{R^{2}} T\right) \ll g_{\mathrm{YM}}^{2} \Delta^{4} \square_{g} T$, so that we can neglect the $\kappa$ contribution (except possibly for the longest "cosmological" scales). Then we can formally solve this equation as

$$
k_{\mu \nu}=\frac{g_{\mathrm{YM}}^{2} \Delta^{4}}{3}\left(-1+\frac{\alpha\left(\alpha+\frac{3}{2}\right)}{3 L_{R}^{2} \square_{g}+\alpha^{2}}\right) T_{\mu \nu} .
$$

Inserting this in the equation for $h_{\mu \nu}$ gives

$$
\square_{g} h_{\mu \nu}=\left(\alpha+\frac{3}{2}\right) \frac{g_{\mathrm{YM}}^{2} \Delta^{4}}{3 L_{R}^{2}}\left(T_{\mu \nu}-\frac{\alpha^{2}}{3 L_{R}^{2} \square_{g}+\alpha^{2}} T_{\mu \nu}\right) .
$$

These two equations describe the emergent gravity on $\mathcal{S}_{\Lambda}^{4}$ (assuming $L_{R} \ll R$ so that $\kappa$ can be neglected). The first equation suggests to separate $k_{\mu \nu}$ into a "local" and a propagating "gravitational" part,

$$
\begin{aligned}
k_{\mu \nu} & =k_{\mu \nu}^{\text {(loc) }}+k_{\mu \nu}^{\text {(grav) }}, \\
k_{\mu \nu}^{(\text {loc })} & =-\frac{g_{\mathrm{YM}}^{2} \Delta^{4}}{3} T_{\mu \nu}, \quad k_{\mu \nu}^{\text {(grav) }}=\left(\alpha+\frac{3}{2}\right) \frac{g_{\mathrm{YM}}^{2} \Delta^{4}}{9 L_{R}^{2}} \frac{\alpha}{\square_{g}+\frac{\alpha^{2}}{3 L_{R}^{2}}} T_{\mu \nu .} .
\end{aligned}
$$

and the effective gravitational metric

$$
\tilde{h}_{\mu \nu}^{\text {(grav) }}:=h_{\mu \nu}+k_{\mu \nu}^{\text {(grav) }}
$$

Now we consider two scaling regimes: 
Regime G: $L_{R}^{2} \square_{g} \gg \alpha^{2}$, i.e. the size of the matter source $T_{\mu \nu}$ is much smaller than $L_{R}^{2} / \alpha^{2}$. This is the "gravity" regime, since then the eom for $h_{\mu \nu}$ and $k_{\mu \nu}^{\text {(grav) }}$ reduce to

$$
\begin{aligned}
\square_{g} h_{\mu \nu} & =\left(\alpha+\frac{3}{2}\right) \frac{g_{\mathrm{YM}}^{2} \Delta^{4}}{3 L_{R}^{2}} T_{\mu \nu} \\
\square_{g} k_{\mu \nu}^{\text {(grav) }} & =\frac{\alpha}{3}\left(\alpha+\frac{3}{2}\right) \frac{g_{\mathrm{YM}}^{2} \Delta^{4}}{3 L_{R}^{2}} T_{\mu \nu}
\end{aligned}
$$

and the effective gravitational metric satisfies

$$
\square_{g} \tilde{h}_{\mu \nu}^{\text {(grav) }}=\left(\frac{\alpha}{3}+1\right)\left(\frac{\alpha}{3}+\frac{1}{2}\right) \frac{g_{\mathrm{YM}}^{2} \Delta^{4}}{L_{R}^{2}} T_{\mu \nu}
$$

We will see that these are indeed the linearized Einstein equations. $k_{\mu \nu}^{(\text {loc })}$ is a small, additional metric contribution with little physical significance. Note that both equations are compatible with the Lorentz gauge condition $\partial^{\mu} h_{\mu \nu}=0=\partial^{\mu} k_{\mu \nu}$. For $\alpha \rightarrow 0$, this regime always applies.

Regime C: $L_{R}^{2} \square_{g r} \ll \alpha^{2}$, i.e. momentum scales much smaller than $\frac{\alpha^{2}}{L_{R}^{2}}$. This is the "cosmological" regime. Then (5.18) essentially reduces to

$$
k_{\mu \nu}=\frac{g_{\mathrm{YM}}^{2} \Delta^{4}}{2 \alpha} T_{\mu \nu}
$$

i.e. $k_{\mu \nu}$ is not propagating at all, and the eom for $h_{\mu \nu}$ also becomes trivial to leading order,

$$
\square_{g} h_{\mu \nu} \approx 0 \text {. }
$$

Hence gravity ceases to operate for structures much larger than $L_{R} / \alpha$.

To understand the intermediate regime, we can formally assign to $h_{\mu \nu}$ the following (negative) gravitational energy-momentum tensor

$$
T_{\mu \nu}[h]:=-\frac{\alpha^{2}}{3 L_{R}^{2} \square_{g}+\alpha^{2}} T_{\mu \nu}
$$

Then (5.20) can be written as

$$
\square_{g} h_{\mu \nu}=\left(\alpha+\frac{3}{2}\right) \frac{g_{\mathrm{YM}}^{2} \Delta^{4}}{3 L_{R}^{2}}\left(T_{\mu \nu}+T_{\mu \nu}[h]\right)
$$

This means that as long as the matter source is sufficiently small, $h_{\mu \nu}$ behaves like an ordinary gravitational field generated by matter; however, for very large sources $T_{\mu \nu}[h]$ becomes significant and shields the effect of gravity.

Self-dual action. We conclude this section with the following interesting observation. Suppose the matrix model has an additional $\epsilon$ term such that it reduces to a selfdual Yang-Mills action ${ }^{15}$

$$
\Gamma_{\mathrm{SD}}\left[\mathcal{F}^{2}\right]=\int d^{4} x\left[\mathcal{F}_{+}^{\mu \nu}(\xi) \mathcal{F}_{\mu \nu}^{+}(\xi)\right]_{0}
$$

\footnotetext{
${ }^{15}$ It was found in $[7,8]$ that the self-dual action indeed arises upon taking into account the volume factor contributed by the fluctuations, cf. (4.33). We leave this possibility for future work.
} 
where

$$
\left[\mathcal{F}_{+}^{\mu \nu} \mathcal{F}_{\mu \nu}^{+}\right]_{0}=\left[2 \mathcal{F}^{\mu \nu} \mathcal{F}_{\mu \nu}+\mathcal{F}^{\mu \nu} \mathcal{F}^{\rho \sigma} \epsilon_{\mu \nu \rho \sigma}\right]_{0}
$$

is averaged over the local fiber, and

$$
\mathcal{F}^{\mu \nu}=\theta^{\mu \mu^{\prime}} A_{\mu^{\prime} \nu^{\prime}} g^{\nu^{\prime} \nu}-\theta^{\nu \nu^{\prime}} A_{\nu^{\prime} \mu^{\prime}} g^{\mu^{\prime} \mu}+\theta^{\mu \mu^{\prime}} \theta^{\nu \nu^{\prime}} F_{\mu^{\prime} \nu^{\prime}}[\mathbf{A}]
$$

cf. (4.30). The first term is equivalent (modulo gauge fixing) to the quadratic action (5.9). The second term $\mathcal{F}^{\mu \nu} \mathcal{F}^{\rho \sigma} \epsilon_{\mu \nu \rho \sigma}$ as usual will not affect the local field equations, except for the mixed term between $A_{\mu \nu}$ and $F_{\mu \nu}$, which after some algebra is

$$
F_{\mu^{\prime} \nu^{\prime}}[\mathbf{A}] \theta^{\mu \mu^{\prime}} \theta^{\nu \nu^{\prime}} \epsilon_{\mu \nu \rho \sigma}\left(\theta^{\rho \rho^{\prime}} A_{\rho^{\prime} \sigma^{\prime}} g^{\sigma^{\prime} \sigma}-\theta^{\sigma \sigma^{\prime}} A_{\sigma^{\prime} \rho^{\prime}} g^{\rho^{\prime} \rho}\right)=-\frac{16 R^{4} r^{2}}{3} \partial^{\mu} \tilde{A}_{\alpha \mu \rho} A^{\rho \alpha}
$$

using selfduality of $A_{\nu \alpha \beta}$, its symmetry property and $\operatorname{det} \theta=\left(\frac{\Delta^{4}}{4}\right)^{2}$. This has indeed the same form as the mixed term in (5.9) (which is the reason for introducing the factor $\alpha$ in (5.9)). Similarly,

$$
F_{\mu^{\prime} \nu^{\prime}}[\mathbf{A}] \theta^{\mu \mu^{\prime}} \theta^{\nu \nu^{\prime}} g_{\mu \rho} g_{\nu \sigma}\left(\theta^{\rho \rho^{\prime}} A_{\rho^{\prime} \sigma^{\prime}} g^{\sigma^{\prime} \sigma}-\theta^{\sigma \sigma^{\prime}} A_{\sigma^{\prime} \rho^{\prime}} g^{\rho^{\prime} \rho}\right) \sim \frac{8 R^{4} r^{2}}{3} \partial^{\mu} \tilde{A}_{\rho \mu \alpha}\left(A^{\rho \alpha}+\frac{\kappa}{R} g^{\rho \alpha}\right)
$$

using partial integration and the gauge fixing condition. The contribution from $h_{\rho \alpha}$ agrees up to a factor (-2) with (5.32). Thus the mixed term cancels in the selfdual Yang-Mills action, which reduces to (5.9) with $\alpha=0$. Then the regime $\mathrm{G}$ always applies, without the need for large $L_{R}$. Together with the following section this implies the linearized Einstein equations always arise, without IR modification. We leave this as an interesting observation.

\subsection{Curvature and linearized Einstein equations}

Now we consider the curvature of the linearized effective metric

$$
G^{\mu \nu}=g^{\mu \nu}+H^{\mu \nu}=\delta^{\mu \nu}+\delta g^{\mu \nu}+H^{\mu \nu}
$$

viewed as a perturbation of the flat metric $\left.\delta^{\mu \nu}\right|_{p}=\left.g^{\mu \nu}\right|_{p}$ near $p$; recall that $H^{\mu \nu}$ was defined in (4.26) as trace-reverse of $\tilde{h}_{\mu \nu}=h_{\mu \nu}+k_{\mu \nu}$.

The first important observation is that the local contribution $k_{\mu \nu}^{\text {(loc) }}(5.21)$ can be dropped; this is merely a negligible local "contact" contribution to the metric, and has nothing to do with any long-distance gravitational effect. For example, to compute the gravitational effect of the sun at the location of the earth, we certainly have $k_{\mu \nu}^{(\text {loc })}=0$. Even for an observer located in a cloud of gas with some energy-momentum density $T_{\mu \nu}$, the "local" contribution $k_{\mu \nu}^{(\text {loc })}=O\left(g_{\mathrm{YM}}^{2} \Delta^{4} T_{\mu \nu}\right)$ would still be negligible except for extremely high energy densities. Thus we will replace $\tilde{h}_{\mu \nu}$ by $\tilde{h}_{\mu \nu}^{\text {(grav) }}(5.22)$, which makes things much more transparent.

Furthermore, assume that we are in the scaling regime G, studying the gravity generated by objects of size $\ll L_{R}^{2}$. Then consider the linearized Ricci tensor [44]

$$
R^{\mu \nu}[g+H] \approx R^{\mu \nu}[g]+\frac{1}{2} \partial^{\mu} \partial^{\nu} H+\frac{1}{2} \partial^{\alpha} \partial_{\alpha} H^{\mu \nu}-\partial^{(\mu} \partial_{\rho} H^{\nu) \rho} .
$$


Since $H_{\mu \nu}$ satisfies the de Donder gauge (4.27), it simplifies as

$$
R_{\mu \nu}[g+H] \approx \frac{3}{R^{2}} g_{\mu \nu}+\frac{1}{2} \partial^{\alpha} \partial_{\alpha} H_{\mu \nu},
$$

where $R_{\mu \nu}[g]=\frac{3}{R^{2}} g_{\mu \nu}$ is the Ricci tensor of $S^{4}$. Hence the linearized Einstein tensor is

$$
\mathcal{G}_{\mu \nu}[g+H]=R_{\mu \nu}[g+H]-\frac{1}{2} g_{\mu \nu} R[g+H] \approx \frac{1}{2} \square_{g} \tilde{h}_{\mu \nu}^{\text {(grav) }}
$$

dropping the curvature contribution $\mathcal{G}[g]=-\frac{3}{R^{2}} g_{\mu \nu}$ of $S^{4}$. Taking into account the equation of motion (5.24) for $\tilde{h}_{\mu \nu}^{\text {(grav) }}$, we obtain the linearized Einstein equations

$$
\mathcal{G}_{\mu \nu}=8 \pi G_{N} T_{\mu \nu}
$$

with the Newton constant given by

$$
G_{N}=\left(\frac{\alpha}{3}+1\right)\left(\frac{\alpha}{3}+\frac{1}{2}\right) \frac{g_{\mathrm{YM}}^{2} \Delta^{4}}{4 \pi L_{R}^{2}}=: L_{p l}^{2} \leq O\left(g_{\mathrm{YM}}^{2} \Delta^{2}\right)
$$

using (2.38). Hence the Planck scale is less than or equal to the uncertainty scale, as expected. As explained above, these equations no longer apply for objects (or rather wavelengths) larger than $L_{R} / \alpha$, where the metric is non-propagating and proportional to $T_{\mu \nu}$ as in regime C. (5.38) entails the interesting reciprocity relation

$$
L_{p l}^{2} L_{R}^{2}=O\left(g_{\mathrm{YM}}^{2} \Delta^{4}\right) .
$$

Since the Einstein equation applies only for wavelengths smaller than $L_{R} / \alpha$, we should require $L_{p l}^{2} \ll L_{R}^{2} / \alpha$. This holds if either of the following conditions is met

$$
c_{n} \gg \sqrt{N} \quad \text { or } \quad \alpha \approx 0 .
$$

Thus we need either a self-dual action, or a "thick" fuzzy sphere $\mathcal{S}_{\Lambda}^{4}$. The latter is easily compatible with $c_{n} \ll N$, such that $L_{R} \ll R$. Notice that such an apparent macroscopic "thickness" $L_{R}$ of $\mathcal{S}_{\Lambda}^{4}$ does not necessarily mean that space is effectively 5-dimensional. This point was discussed in section 4.1, although a more detailed investigation is required to settle this. There is no issue with dimensional reduction for the self-dual action (5.29), where $L_{R}$ does not act as a IR cutoff, so that $c_{n}$ can be very small.

Now consider briefly the gravitational coupling of fermions. We only observe here that the matrix model Dirac operator

$$
\not D \Psi=\Gamma_{a}\left[X^{a}, \Psi\right] \sim i\left(\tilde{\gamma}^{\mu} \partial_{\mu}+\ldots\right) \Psi
$$

can be rewritten in terms of "comoving" Clifford generators $\tilde{\gamma}^{\mu}=\Gamma_{\alpha} \tilde{e}^{\alpha \mu}$ (up to possibly a conformal factor), which encode the effective metric $\left\{\tilde{\gamma}^{\mu}, \tilde{\gamma}^{\nu}\right\}=2 G^{\mu \nu}$ [8]. Together with supersymmetry [1], we expect that fermions couple properly to gravity in the present framework, however a detailed analysis is left for future work.

The above results show that the 4-dimensional (Euclidean, linearized) Einstein equations can emerge from the classical dynamics of fluctuations on fuzzy 4 -spheres $\mathcal{S}_{\Lambda}^{4}$ in the 
Yang-Mills matrix model (3.1), provided certain scaling conditions for $\Lambda$ are met, and dimensional reduction is justified. No explicit Einstein-Hilbert term is required. There are several contributions to the metric fluctuation $\tilde{h}_{\mu \nu}$, so that the physics is richer than in general relativity. Most notably there is a long-distance modification, in the sense that wavelengths much larger than $L_{R}$ do not contribute to gravity. The requirement of large $L_{R}$ (i.e. large $c_{n}$ ) is avoided for the self-dual action.

In any case, the long-wavelength modifications of gravity discussed above should be very interesting for cosmology, and it is tempting to relate this to some of the effects attributed to dark matter or dark energy. There will also be some additional gravitational modes arising e.g. from radial deformations $\kappa$. However, a more detailed analysis is required before these issues can be addressed.

One final but crucial question is whether the present mechanism survives quantization. The (preliminary and partial) analysis in the following section supports the conjecture that the quantization is well-behaved and preserves the above picture, for the IKKT model.

\section{One-loop corrections from string states}

As a first step towards a full quantum theory, we would like to study the one-loop effective action for the above gravitational fluctuations around a fuzzy $\mathcal{S}_{\Lambda}^{4}$ background. This should be done in the maximally supersymmetric IIB or IKKT model, which is the only model where the non-local UV/IR mixing in noncommutative field theory is mild (leading to 10dimensional IIB supergravity in target space). Until recently, such a 1-loop computation in terms of a mode expansion would be hopeless; already the one-loop effective action for the constant radius is quite involved [36]. However the integration method using string states [25] makes this task feasible. As a check of these methods we will first reproduce the results in [36], and then proceed to extract the leading 1-loop contributions to the effective action. For simplicity we will restrict ourselves to the basic fuzzy sphere $\mathcal{S}_{N}^{4}$ here.

\subsection{The 1-loop effective potential for the IKKT model}

We start with the bare bosonic action (3.1) for the background $X$

$$
S_{0}[X]=\frac{1}{g^{2}} \operatorname{Tr}\left(-\left[X_{i}, X_{j}\right]\left[X^{i}, X^{j}\right]+\mu^{2} X^{i} X_{i}\right)
$$

supplemented by a mass $\mu^{2}$, to regularize possible IR singularities. Adding the fermions in the IKKT model, the one-loop effective action is defined by

$$
Z[r, \mu]=\int_{1 \text { loop }} d X d \Psi e^{-S[r \bar{X}, \Psi]}=e^{-\Gamma_{\text {eff }}[r, \mu]}
$$

and we will write

$$
\Gamma_{\text {eff }}[r, \mu]=S_{0}[X]+\Gamma_{1 \text { loop }}[r, \mu]
$$


We recall the following form of the one-loop effective action in the IKKT model $[1,37,45]$

$$
\begin{aligned}
\Gamma_{\text {1loop }}[X]= & \frac{1}{2} \operatorname{Tr}\left(\log \left(\square+\frac{\mu^{2}}{2}-M_{a b}^{(\mathcal{A})}\left[\Theta^{a b}, .\right]\right)-\frac{1}{2} \log \left(\square-M_{a b}^{(\psi)}\left[\Theta^{a b}, .\right]\right)-2 \log (\square)\right) \\
= & \frac{1}{2} \operatorname{Tr}\left(\sum_{n>0} \frac{1}{n}\left(\left(\square^{-1}\left(-M_{a b}^{(\mathcal{A})}\left[\Theta^{a b}, .\right]+\frac{1}{2} \mu^{2}\right)\right)^{n}-\frac{1}{2}\left(-\square^{-1} M_{a b}^{(\psi)}\left[\Theta^{a b}, .\right]\right)^{n}\right)\right) \\
= & \frac{1}{2} \operatorname{Tr}\left(\frac{1}{4} \square^{-1}\left(M_{a b}^{(\mathcal{A})}\left[\Theta^{a b}, .\right]\right)^{4}-\frac{1}{8}\left(\square^{-1} M_{a b}^{(\psi)}\left[\Theta^{a b}, .\right]\right)^{4}+\mathcal{O}\left(\square^{-1}\left[\Theta^{a b}, .\right]\right)^{5}\right) \\
& +\frac{1}{4} \mu^{2} \operatorname{Tr} \square^{-1}+O\left(\mu^{4}\right)
\end{aligned}
$$

with $a, b=1, \ldots, 10$, where

$$
\begin{aligned}
r l\left(M_{a b}^{(\psi)}\right)_{\beta}^{\alpha} & =\frac{1}{4 i}\left[\Gamma_{a}, \Gamma_{b}\right]_{\beta}^{\alpha} \\
\left(M_{a b}^{(\mathcal{A})}\right)_{d}^{c} & =i\left(\delta_{b}^{c} \delta_{a d}-\delta_{a}^{c} \delta_{b d}\right),
\end{aligned}
$$

and the $2 \log \square$ term arises from the ghost contribution. Note that the coupling constant $g$ drops out from $\Gamma_{1 \text { loop }}$ due to supersymmetry. For $\mu=0$, the first non-vanishing term in this expansion is $n=4$ due to maximal supersymmetry. However there are contributions of order $\Theta$ for $\mu^{2} \neq 0$ due to the soft SUSY breaking, which are important to stabilize the background.

The leading 4 th order term is given by the following expression [37]:

$$
\begin{aligned}
\Gamma_{\text {loop } ; 4}[X]= & \frac{1}{8} \operatorname{Tr}\left(\left(\square^{-1}\left(M_{a b}^{(\mathcal{A})}\left[\Theta^{a b}, .\right]\right)^{4}-\frac{1}{2}\left(\square^{-1} M_{a b}^{(\psi)}\left[\Theta^{a b}, .\right]\right)^{4}\right)\right. \\
= & \left.\left.\frac{1}{4} \operatorname{Tr}\left(\square^{-1}\left[\Theta^{a_{1} b_{1}}, \ldots \square^{-1}\left[\Theta^{a_{4} b_{4}}, .\right]\right]\right]\right]\right) \\
& \left(-4 g_{b_{1} a_{2}} g_{b_{2} a_{3}} g_{b_{3} a_{4}} g_{b_{4} a_{1}}-4 g_{b_{1} a_{2}} g_{b_{2} a_{4}} g_{b_{4} a_{3}} g_{b_{3} a_{1}}-4 g_{b_{1} a_{3}} g_{b_{3} a_{2}} g_{b_{2} a_{4}} g_{b_{4} a_{1}}\right. \\
& \left.+g_{b_{1} a_{2}} g_{b_{2} a_{1}} g_{b_{3} a_{4}} g_{b_{4} a_{3}}+g_{b_{1} a_{3}} g_{b_{3} a_{1}} g_{b_{2} a_{4}} g_{b_{4} a_{2}}+g_{b_{1} a_{4}} g_{b_{4} a_{1}} g_{b_{2} a_{3}} g_{b_{3} a_{2}}\right)
\end{aligned}
$$

and the leading term in $\mu^{2}$ is

$$
\Gamma_{1 \text { loop }}\left[X ; \mu^{2}\right]=-\frac{1}{4} \mu^{2} \operatorname{Tr}\left(\square^{-1}\right) .
$$

We will evaluate the trace over hermitian matrices in $\operatorname{End}(\mathcal{H})$ using the basic formula [25]

$$
\operatorname{Tr}_{E n d(\mathcal{H})} \mathcal{O}=\frac{(\operatorname{dim} \mathcal{H})^{2}}{(\operatorname{Vol} \mathcal{M})^{2}} \int_{\mathcal{M} \times \mathcal{M}} d \mathbf{x} d \mathbf{y}(|\mathbf{x}\rangle\langle\mathbf{y}|) \mathcal{O}(|\mathbf{y}\rangle\langle\mathbf{x}|)
$$

Here $|\mathbf{y}\rangle\langle\mathbf{x}| \in \operatorname{End}(\mathcal{H})$ are string states, ${ }^{16}$ which are built out of coherent states $|\mathbf{x}\rangle$ on $\mathcal{M}=\mathbb{C} P^{3} \approx \mathcal{S}_{N}^{4} \times S^{2}$. The formula is exact for homogeneous spaces such as $\mathbb{C} P^{3}$. It follows by noting that the r.h.s. of (6.8) is invariant under $\mathrm{SO}(5)_{L} \times \mathrm{SO}(5)_{R}$, and so is the

\footnotetext{
${ }^{16}$ Bold face letters $\mathbf{x}, \mathbf{y}, \ldots$ denote points in $\mathbb{C} P^{3}$, while plain letters $x, y, \ldots$ denote their projection on $S^{4}$.
} 
trace functional on $\operatorname{End}\left(\mathcal{H}_{\Lambda}\right)$. The normalization of the measure ${ }^{17}$ in the integrals cancels out, and we will choose it as product measure on $S^{2} \times S^{4}$ with unit volume of $S^{2}$ and the measure on $S^{4}$ is induced by the target space metric. For deformed $\mathcal{M},(6.8)$ is expected to be an excellent approximation, as long as $\mathcal{O}$ is well localized.

The string states are very useful for loop computations, because they have approximate localization properties in both position and momentum; see [25] for a detailed discussion. In particular,

$$
\begin{aligned}
\square^{-1}(|\mathbf{y}\rangle\langle\mathbf{x}|) & \sim \frac{1}{|x-y|^{2}+2 \Delta^{2}}|\mathbf{y}\rangle\langle\mathbf{x}| \\
\square^{-1}\left[\Theta^{a b}, .\right](|\mathbf{y}\rangle\langle\mathbf{x}|) & \sim \frac{1}{|x-y|^{2}+2 \Delta^{2}} \delta \Theta^{a b}(\mathbf{y}, \mathbf{x})|\mathbf{y}\rangle\langle\mathbf{x}| \\
\delta \Theta^{a b}(\mathbf{y}, \mathbf{x}) & =\Theta^{a b}(\mathbf{y})-\Theta^{a b}(\mathbf{x}) .
\end{aligned}
$$

We can therefore approximately evaluate the 1-loop integral as follows

$$
\begin{aligned}
& \Gamma_{\text {lloop } ; 4}[X]=\frac{1}{4} \frac{(\operatorname{dim} \mathcal{H})^{2}}{(\operatorname{Vol}(\mathcal{M}))^{2}} \int_{\mathcal{M} \times \mathcal{M}} d \mathbf{x} d \mathbf{y} \frac{\delta \Theta^{a_{1} b_{1}}(\mathbf{y}, \mathbf{x}) \delta \Theta^{a_{2} b_{2}}(\mathbf{y}, \mathbf{x}) \delta \Theta^{a_{3} b_{3}}(\mathbf{y}, \mathbf{x}) \delta \Theta^{a_{4} b_{4}}(\mathbf{y}, \mathbf{x})}{\left(|x-y|^{2}+2 \Delta^{2}\right)^{4}} \\
& 3\left(-4 g_{b_{1} a_{2}} g_{b_{2} a_{3}} g_{b_{3} a_{4}} g_{b_{4} a_{1}}+g_{b_{1} a_{2}} g_{b_{2} a_{1}} g_{b_{3} a_{4}} g_{b_{4} a_{3}}\right) \\
& =\frac{1}{4} \frac{(\operatorname{dim} \mathcal{H})^{2}}{(\operatorname{Vol}(\mathcal{M}))^{2}} \int_{\mathcal{M} \times \mathcal{M}} d \mathbf{x} d \mathbf{y} \frac{3 S_{4}[\delta \Theta(\mathbf{x}, \mathbf{y})]}{\left(|x-y|^{2}+2 \Delta^{2}\right)^{4}} \\
& \Gamma_{\text {1loop }}\left[X ; \mu^{2}\right]=\frac{5}{2} \frac{(\operatorname{dim} \mathcal{H})^{2}}{(\operatorname{Vol}(\mathcal{M}))^{2}} \int_{\mathcal{M} \times \mathcal{M}} d \mathbf{x} d \mathbf{y} \frac{\mu^{2}}{|x-y|^{2}+2 \Delta^{2}}
\end{aligned}
$$

where

$$
S_{4}[\delta \Theta]=-4 \operatorname{tr} \delta \Theta^{4}+\left(\operatorname{tr} \delta \Theta^{2}\right)^{2}
$$

(suppressing the target space metric $g_{a b}$ ). First, we note that $\Gamma_{1 \mathrm{loop} ; 4}[X]$ vanishes identically for constant fluxes $\Theta=$ const. This is a reflection of the maximal supersymmetry of such backgrounds. Due to the SUSY cancellations, the interaction decays like $r^{-8}$, and it is bounded at short distances by the NC cutoff $\Delta^{2}$. This means that the one-loop induced action is a weak short-distance effect on branes with dimension less than 10 (which is essentially IIB supergravity). We will compute its effect on the fluctuations on the fuzzy $S^{4}$ background below.

The following observation [46] is very useful: if $\delta \Theta^{a b}(\mathbf{y}, \mathbf{x})$ has rank $\leq 4$ for any fixed points $\mathbf{y}, \mathbf{x}$ (which holds for any geometries embedded in $\mathbb{R}^{5}$ ), then

$$
\begin{aligned}
-S_{4}[\delta \Theta] & =4 \operatorname{tr}(\delta \Theta g \delta \Theta g \delta \Theta g \delta \Theta g)-(\operatorname{tr} \delta \Theta g \delta \Theta g)^{2} \\
& =4\left(\delta \Theta_{+}^{a b} \delta \Theta_{+b a}\right)\left(\delta \Theta_{-}^{c d} \delta \Theta_{-d c}\right), \quad \delta \Theta_{ \pm}=\delta \Theta \pm \star_{g} \delta \Theta \\
& \geq 0
\end{aligned}
$$

where $\star_{g}$ denotes the 4 -dimensional Hodge star with respect to $g_{\mu \nu}$. Hence $S_{4}$ leads to an attractive interaction, which vanishes only in the (anti-) selfdual case $\delta \Theta= \pm \star_{g} \delta \Theta$. This means that the quantum effects on fuzzy $S^{4}$ are small, because $\theta^{\mu \nu}$ is self-dual here.

\footnotetext{
${ }^{17}$ The proper measure is the symplectic volume form on the underlying $\mathbb{C} P^{3}$.
} 


\subsection{Vacuum energy of $\mathcal{S}_{N}^{4}$}

Mass contribution. We start with the contribution of $\mu^{2}(6.10)$ :

$$
\begin{aligned}
\Gamma_{\text {1loop }}\left[X ; \mu^{2}\right] & =\frac{5}{2} \frac{(\operatorname{dim} \mathcal{H})^{2}}{(\operatorname{Vol}(\mathcal{M}))^{2}} \int_{\mathcal{M} \times \mathcal{M}} d \mathbf{x} d \mathbf{y} \frac{\mu^{2}}{|x-y|^{2}+2 \Delta^{2}} \\
& =\frac{5}{2} \frac{(\operatorname{dim} \mathcal{H})^{2}}{\operatorname{Vol}\left(\mathrm{S}^{4}\right)} \int_{S^{4}} d x \frac{\mu^{2}}{R^{2}|x-p|^{2}+2 \Delta^{2}} \\
& =\mu^{2} \frac{5}{2} \frac{(\operatorname{dim} \mathcal{H})^{2}}{R^{2} \frac{8 \pi^{2}}{3}} \int_{0}^{\pi} 2 \pi^{2} d \vartheta \frac{\sin ^{3} \vartheta}{(1-\cos \vartheta)^{2}+\sin \vartheta^{2}+2 \tilde{\Delta}^{2}} \\
& =\frac{\mu^{2}}{r^{2}} \frac{15}{8} \frac{(\operatorname{dim} \mathcal{H})^{2}}{R_{N}^{2}}\left(1+O\left(\tilde{\Delta}^{2}\right)\right)
\end{aligned}
$$

where

$$
\tilde{\Delta}^{2} \sim \frac{\Delta^{2}}{R^{2}}=\frac{4}{N}
$$

using (2.18). Note that $S^{4}$ denotes the unit sphere in this computation. Using

$$
\operatorname{dim} \mathcal{H}=\frac{1}{6}(N+1)(N+2)(N+3),
$$

we obtain

$$
\Gamma_{1 \text { loop }}\left[X ; \mu^{2}\right]=\frac{5}{24} N^{4} \frac{\mu^{2}}{r^{2}}\left(1+O\left(\frac{1}{N}\right)\right) .
$$

This agrees precisely with the group-theoretical computation in [36]. This term describes the positive vacuum energy contribution due to the explicit SUSY breaking by the bosonic mass $\mu^{2}$, which scales like $\frac{\mu^{2}}{r^{2}}$.

Curvature contribution. Now we compute the $\mu^{2}=0$ contribution

$$
\Gamma_{1 \text { loop } ; 4}[X]=\frac{1}{4} \frac{(\operatorname{dim} \mathcal{H})^{2}}{(\operatorname{Vol}(\mathcal{M}))^{2}} \int_{\mathcal{M} \times \mathcal{M}} d \mathbf{x} d \mathbf{y} \frac{3 S_{4}[\delta \Theta(\mathbf{x}, \mathbf{y})]}{\left(|x-y|^{2}+2 \Delta^{2}\right)^{4}}
$$

We can fix $\mathbf{x}=(x, \xi)$ to be some fixed reference point on $\mathcal{M} \approx S^{4} \times S^{2}$, where $x^{\mu}$ are local coordinates on $S^{4}$. We first compute the integration over $\eta \in S^{2}$ with $y=x$, which is the projection defined in $(2.25)$

$$
[f(x, \eta)]_{S^{2}}:=\frac{1}{4 \pi} \int_{S^{2}} d \eta f(x, \eta)
$$


at any given $x \in S^{4}$. Recalling the identity $\gamma^{b c}=\frac{1}{4} \Delta^{4} P_{T}^{b c}(x)$ where $P_{T}$ is the tangential projector on $S^{4} \subset \mathbb{R}^{5}$ and using (C.1), this gives

$$
\begin{aligned}
{\left[\theta_{\mathbf{x}}^{a b} \theta_{\mathbf{x}}^{c d}\right]_{S^{2}} } & =\frac{1}{12} \Delta^{4}\left(P_{T}^{a c}(x) P_{T}^{b d}(x)-P_{T}^{b c}(x) P_{T}^{a d}(x)+\varepsilon^{a b c d e} x^{e}\right) \\
{\left[\gamma_{\mathbf{x}}^{a b} \gamma_{\mathbf{y}}^{b c}\right]_{S^{2} \times S^{2}} } & =\frac{1}{16} \Delta^{8}\left(g^{a c}-y^{a} y^{c}-x^{a} x^{c}+x^{a}(x \cdot y) y^{c}\right) \\
{\left[\operatorname{tr} \gamma_{\mathbf{x}} \gamma_{\mathbf{y}}\right]_{S^{2} \times S^{2}} } & =\frac{1}{16} \Delta^{8} P_{T}^{a b}(x) P_{T}^{b a}(y)=\frac{1}{16} \Delta^{8}\left(3+(x \cdot y)^{2}\right) \\
{\left[\operatorname{tr}\left(\theta_{\mathbf{x}} \theta_{\mathbf{y}} \theta_{\mathbf{x}} \theta_{\mathbf{y}}\right)\right]_{S^{2} \times S^{2}} } & =\frac{1}{144} \Delta^{8}\left(\left(3+(x \cdot y)^{2}\right)\left(2+(x \cdot y)^{2}\right)-24(x \cdot y)\right) \stackrel{x \rightarrow y}{\rightarrow}-\frac{1}{12} \Delta^{8} \\
{\left[\left(\operatorname{tr}\left(\theta_{\mathbf{x}} \theta_{\mathbf{y}}\right)\right)^{2}\right]_{S^{2} \times S^{2}} } & =\frac{1}{144} \Delta^{8}\left(2\left(3+(x \cdot y)^{2}\right)\left(2+(x \cdot y)^{2}\right)+24(x \cdot y)\right) \stackrel{x \rightarrow y}{\rightarrow} \frac{1}{3} \Delta^{8}
\end{aligned}
$$

using the notation $\theta_{\mathbf{x}}^{a b}=\theta^{a b}(x, \eta)$ etc., and $x \cdot y=x^{b} y_{b}$, and

$$
\begin{aligned}
P_{T}^{a b}(x) P_{T}^{a b}(y) & =3+(x \cdot y)^{2} \\
P_{T}^{a b}(x) P_{T}^{b c}(y) P_{T}^{c d}(x) P_{T}^{d a}(y) & =3+(x \cdot y)^{2} \\
\varepsilon^{a b c d e} x_{e} \varepsilon_{a b c d f} y^{f} & =24(x \cdot y) .
\end{aligned}
$$

We can now evaluate (6.17) as follows

$$
\begin{aligned}
\Gamma_{1 \text { loop } ; 4}[X]= & \frac{3}{4} \frac{(\operatorname{dim} \mathcal{H})^{2}}{(\operatorname{Vol}(\mathcal{M}))^{2}} \int_{\mathcal{M} \times \mathcal{M}} d \mathbf{x} d \mathbf{y} \frac{1}{\left(|x-y|^{2}+2 \Delta^{2}\right)^{4}}\left[-4 \operatorname{tr}\left(\gamma_{\mathbf{x}}^{2}\right)+\left(\operatorname{tr} \gamma_{\mathbf{x}}\right)^{2}+(\mathbf{x} \leftrightarrow \mathbf{y})\right. \\
& +4\left(4 \operatorname{tr}\left(\gamma_{\mathbf{x}} \theta_{\mathbf{x}} \theta_{\mathbf{y}}\right)-\operatorname{tr}\left(\theta_{\mathbf{x}} \theta_{\mathbf{y}}\right) \operatorname{tr}\left(\gamma_{\mathbf{x}}\right)+(\mathbf{x} \leftrightarrow \mathbf{y})\right) \\
& \left.-16 \operatorname{tr}\left(\gamma_{\mathbf{x}} \gamma_{\mathbf{y}}\right)+2 \operatorname{tr} \gamma_{\mathbf{x}} \operatorname{tr} \gamma_{\mathbf{y}}-8 \operatorname{tr}\left(\theta_{\mathbf{x}} \theta_{\mathbf{y}} \theta_{\mathbf{x}} \theta_{\mathbf{y}}\right)+4\left(\operatorname{tr}\left(\theta_{\mathbf{x}} \theta_{\mathbf{y}}\right)\right)^{2}\right]_{S^{2} \times S^{2}} \\
= & -\frac{3}{4} \frac{(\operatorname{dim} \mathcal{H})^{2}}{\left(\operatorname{Vol}\left(\mathrm{S}^{4}\right)\right)^{2}} \frac{\Delta^{8}}{R^{8}} \int_{S^{4} \times S^{4}} d x d y \frac{(1-(x \cdot y))^{2}}{\left(|x-y|^{2}+2 \tilde{\Delta}^{2}\right)^{4}} \\
= & -\frac{1}{4} N^{2}\left(-\frac{17}{3}+2 \ln 2-2 \ln \tilde{\Delta}^{2}+\mathcal{O}\left(\tilde{\Delta}^{2}\right)\right) \\
= & -\frac{1}{2} N^{2}\left(\ln N+O(1)+\mathcal{O}\left(\tilde{\Delta}^{2}\right)\right) .
\end{aligned}
$$

Again the last line agrees with the (more involved) group-theoretical computation in [36], providing further support for the coherent state approach. The present computation is not only shorter, it also allows to see more clearly the origin of the attractive interaction: it arises from nearly-local interaction among the $N$ degenerate sheets at points $x \approx y \in S^{4}$. At coincident locations $x=y$, the cancellation is exact, because $\theta^{\mu \nu}(x, \xi)$ is selfdual. In other words, the interaction is a residual attractive IIB supergravity effect which arises due to the curvature of $S^{4}$. This also confirms the stabilization mechanism put forward in [36].

\subsection{Fluctuations}

Having gained confidence in the coherent state approach to 1-loop integrals on fuzzy $S^{4}$, we turn to the 1-loop effective action for the fluctuation fields. This is of course a major task, and we will only consider the leading corrections to the kinetic terms for the lowest spin excitations of interest here. 


\subsection{Transversal fluctuations}

Consider first the contributions from the transversal flux components $\mathcal{F}^{\mu a} \sim-i\left[X^{\mu}, \mathcal{A}^{a}\right]$ where $a=5, \ldots, 10$. It is easy to see that only the transversal fluctuations $\phi^{i}$ in

$$
X^{a}=\left(\begin{array}{c}
x^{\mu} \\
\phi^{i}
\end{array}\right)
$$

contribute to $\mathcal{F}^{\mu a}$. The general formula (6.12) for $S_{4}[\delta \theta+\delta \mathcal{F}]$ shows that the dominant interactions arise for points $\mathbf{x}=(x, \xi)$ and $\mathbf{y}=(x, \eta)$ on $\mathcal{M}=\mathbb{C} P^{3}$ with the same $x \in S^{4}$. Then transversal fluctuations can arise only at quadratic (or higher) order, contracted as

$$
\begin{aligned}
\delta \mathcal{F}^{\mu a} g_{a b} \delta \mathcal{F}^{b \nu} & =\left(\mathcal{F}^{\mu a}(x, \xi)-\mathcal{F}^{\mu a}(x, \eta)\right) g_{a b}\left(\mathcal{F}^{b \nu}(x, \xi)-\mathcal{F}^{b \nu}(x, \eta)\right) \\
& =\delta \theta^{\mu \alpha} \tilde{T}[\phi]_{\alpha \beta} \delta \theta^{\beta \nu} .
\end{aligned}
$$

Here we assume that $\phi^{a}=\phi^{a}(x)$ is constant along the $S^{2}$ fiber, so that $\mathcal{F}^{\mu a}=\theta^{\mu \alpha} \partial_{\alpha} \phi^{a}(4.6)$, and

$$
\tilde{T}[\phi]_{\alpha \beta}=\partial_{\alpha} \phi^{a} \partial_{\beta} \phi_{a}, \quad \delta \theta^{\mu \alpha}=\theta^{\mu \alpha}(x, \xi)-\theta^{\mu \alpha}(x, \eta) .
$$

However, we claim that this quadratic contribution in $\phi$ cancels due to the averaging over $S^{2}$, and the only non-vanishing contributions are higher-order interactions or higherderivative terms. To see this, note that the quadratic contribution would arise from

$$
\begin{aligned}
S_{4}[\phi]= & -4 \operatorname{tr}\left((\delta \theta+\delta \mathcal{F})^{4}\right)+\left(\operatorname{tr}(\delta \theta+\delta \mathcal{F})^{2}\right)^{2} \\
= & -16 \operatorname{tr}(\delta \theta \delta \theta \delta \theta \delta \theta \tilde{T}[\phi])+4 \operatorname{tr}(\delta \theta \delta \theta) \operatorname{tr}(\delta \theta \delta \theta \tilde{T}[\phi]) \\
& +O\left(\phi^{4}\right)+O\left(\left(\partial^{2} \phi\right)^{2}\right) .
\end{aligned}
$$

Averaging over $(\xi, \eta) \in S^{2} \times S^{2}$ and using invariance under $\mathrm{SU}(2)_{L}$ and therefore under $\mathrm{SO}(4)$ (noting that $\tilde{T}[\phi]$ is constant on $S^{2}$ ) gives

$$
\begin{aligned}
{\left[(\delta \theta \delta \theta \delta \theta \delta \theta)^{\mu \nu}\right]_{S^{2} \times S^{2}} } & =\frac{1}{4} g^{\mu \nu}[\operatorname{tr}(\delta \theta \delta \theta \delta \theta \delta \theta)]_{S^{2} \times S^{2}} \\
{\left[\operatorname{tr}(\delta \theta \delta \theta)(\delta \theta \delta \theta)^{\mu \nu}\right]_{S^{2} \times S^{2}} } & =\frac{1}{4} g^{\mu \nu}[\operatorname{tr}(\delta \theta \delta \theta) \operatorname{tr}(\delta \theta \delta \theta)]_{S^{2} \times S^{2}} .
\end{aligned}
$$

Contracting with $\tilde{T}[\phi]_{\mu \nu}$ and recalling that $S_{4}[\delta \theta]=0$ for the self-dual background, we conclude that

$$
S_{4}[\phi]=0+O\left(\phi^{4}\right)+O\left(\left(\partial^{2} \phi\right)^{2}\right) .
$$

Therefore transversal deformations of the background do not acquire quadratic quantum corrections at one loop, up to possible subleading higher-derivative terms. As a check, $S_{4}[\phi]$ vanishes for radial deformations $A^{a}=X^{a}$, where $\tilde{T}^{\mu \nu} \sim g^{\mu \nu}$. This is in contrast to tangential deformations, as we will see.

\subsection{Tangential fluctuations}

The one-loop effective action is given by

$$
\Gamma_{\text {lloop; } 4}[X]=\frac{1}{4} \frac{(\operatorname{dim} \mathcal{H})^{2}}{\left(\operatorname{Vol}\left(\mathcal{M}^{6}\right)\right)^{2}} \int_{\mathcal{M} \times \mathcal{M}} d \mathbf{x} d \mathbf{y} \frac{3 S_{4}[\delta \theta(\mathbf{x}, \mathbf{y})+\delta \mathcal{F}(\mathbf{x}, \mathbf{y})]}{\left(|x-y|^{2}+2 \Delta^{2}\right)^{4}} .
$$


The propagators act like a short-range delta function with normalization

$$
\int_{\mathcal{M}^{4}} d x \frac{1}{\left(|x-y|^{2}+2 \Delta_{x}^{2}\right)^{4}} \approx \int_{\mathbb{R}^{4}} d^{4} x \frac{1}{\left(|x|^{2}+2 \Delta^{2}\right)^{4}}=\frac{\pi^{2}}{6} \frac{1}{\Delta^{4}}
$$

using the Riemannian measure, and $\mathcal{M}^{4}$ indicates $S^{4}$ with radius $R$. Therefore the dominant contribution will come from local interactions with $\mathbf{x}=(x, \xi)$ and $\mathbf{y}=(x, \xi)$ denoting the same $x \in \mathcal{M}^{4}$ but different points on the internal $S^{2}$. We can therefore replace the integral over $\mathcal{M}^{4} \times \mathcal{M}^{4}$ by a single integral as follows

$$
\Gamma_{\text {1loop } ; 4}[X]=\frac{\pi^{2}}{8} \frac{(\operatorname{dim} \mathcal{H})^{2}}{\left(\operatorname{Vol}\left(\mathcal{M}^{4}\right)\right)^{2}\left(\operatorname{Vol}\left(\mathrm{S}^{2}\right)\right)^{2}} \frac{1}{\Delta^{4}} \int_{\mathcal{M}^{4} \times S^{2} \times S^{2}} d x d \xi d \eta S_{4}[\delta \theta(\mathbf{x}, \mathbf{y})+\delta \mathcal{F}(\mathbf{x}, \mathbf{y})]
$$

For the tangential fluctuations, $S_{4}$ can be written locally using (6.12) in terms of (antiselfdual) flux components as follows:

$$
\begin{aligned}
& S_{4}[\delta \theta+\delta \mathcal{F}]=-4\left(\theta_{+}(\mathbf{x})-\theta_{+}(\mathbf{y})+\mathcal{F}_{+}(\mathbf{x})-\mathcal{F}_{+}(\mathbf{y})\right)^{\mu \nu}\left(\theta_{+}(\mathbf{x})-\theta_{+}(\mathbf{y})+\mathcal{F}_{+}(\mathbf{x})-\mathcal{F}_{+}(\mathbf{y})\right)_{\mu \nu} \\
& \cdot\left(\mathcal{F}_{-}(\mathbf{x})-\mathcal{F}_{-}(\mathbf{y})\right)^{\rho \sigma}\left(\mathcal{F}_{-}(\mathbf{x})-\mathcal{F}_{-}(\mathbf{y})\right)_{\rho \sigma} \\
& \approx-4 m^{2}(\mathbf{x}, \mathbf{y})\left(\mathcal{F}_{-}(\mathbf{x})-\mathcal{F}_{-}(\mathbf{y})\right)^{\rho \sigma}\left(\mathcal{F}_{-}(\mathbf{x})-\mathcal{F}_{-}(\mathbf{y})\right)_{\rho \sigma} \leq 0 \\
& \mathcal{F}_{ \pm}= \mathcal{F} \pm \star_{g} \mathcal{F} \\
& m^{2}(\mathbf{x}, \mathbf{y})=(\theta(\mathbf{x})-\theta(\mathbf{y}))^{\mu \nu}(\theta(\mathbf{x})-\theta(\mathbf{y}))_{\mu \nu} \sim \Delta^{4}\|\xi-\eta\|^{2}>0
\end{aligned}
$$

using (C.5). Here we used the self-duality of the background $\theta_{-}=0$, while $\theta_{+}+\mathcal{F}_{+} \approx \theta_{+}$ for small fluctuations. As above, $\mathbf{x}, \mathbf{y}$ denote the same $x \in \mathcal{M}^{4}$ but different points $\xi, \eta$ on the internal $S^{2}$. This should be integrated over $S^{2} \times S^{2}$ for each $x \in \mathcal{M}^{4}$. Since $m^{2}>0$ whenever $\xi \neq \eta$, only the ASD components $\mathcal{F}_{-}^{\mu \nu}$ contribute, with a negative sign. Hence fluctuations $\mathcal{F}(x) \in(n, 0)$ which are constant along $S^{2}$ drop out, but all the higher spin fluctuations such as $\mathcal{F}(x) \in(n, 2)$ will contribute.

The gravity modes of interest here give rise to $\mathcal{F}^{\mu \nu} \in(n, 2)$. These can be written as

$$
\mathcal{F}^{\mu \nu}(x, \xi)=\mathcal{F}_{a}^{\mu \nu}(x) \xi^{a}
$$

for $\xi_{a} \in S^{2}$ and $\mathcal{F}_{a}^{\mu \nu}$ a 3 -vector. Then

$$
\begin{aligned}
(\mathcal{F}(\xi)-\mathcal{F}(\eta))^{\mu \nu}(\mathcal{F}(\xi)-\mathcal{F}(\eta))_{\mu \nu} & =\mathcal{F}_{a}^{\mu \nu} \mathcal{F}_{\mu \nu}^{b}(\xi-\eta)_{a}(\xi-\eta)^{b} \\
\mathcal{F}_{a}^{\mu \nu} \mathcal{F}_{\mu \nu}^{a} & =3\left[\mathcal{F}^{\mu \nu}(\xi) \mathcal{F}_{\mu \nu}(\xi)\right]_{S^{2}}
\end{aligned}
$$

Using

$$
\frac{1}{\left(\operatorname{Vol} S^{2}\right)^{2}} \int_{S^{2} \times S^{2}}\|\xi-\eta\|^{2}(\xi-\eta)^{a}(\xi-\eta)^{b}=\frac{16}{9} \delta^{a b}
$$

we can write

$$
\frac{1}{\left(\operatorname{Vol} S^{2}\right)^{2}} \int_{S^{2} \times S^{2}}\|\xi-\eta\|^{2}(\mathcal{F}(\xi)-\mathcal{F}(\eta))^{\mu \nu}(\mathcal{F}(\xi)-\mathcal{F}(\eta))_{\mu \nu}=\frac{16}{3}\left[\mathcal{F}^{\mu \nu}(\xi) \mathcal{F}_{\mu \nu}(\xi)\right]_{S^{2}} .
$$


This means that for such $\mathcal{F} \in(n, 2)$, the 1-loop effective action at $O\left(\mathcal{F}^{2}\right)$ can be written as

$$
\Gamma_{1-\text { loop } ; 4}\left[\mathcal{F}^{2}\right]=-\frac{8 \pi^{2}}{3} \frac{(\operatorname{dim} \mathcal{H})^{2}}{\left(\operatorname{Vol} \mathcal{M}^{4}\right)^{2}} \int_{\mathcal{M}^{4}} d x\left[\mathcal{F}_{-}^{\mu \nu}(\xi) \mathcal{F}_{\mu \nu}^{-}(\xi)\right]_{S^{2}}
$$

where

$$
\left[\mathcal{F}_{-}^{\mu \nu} \mathcal{F}_{\mu \nu}^{-}\right]_{S^{2}}=\left[2 \mathcal{F}^{\mu \nu} \mathcal{F}_{\mu \nu}-\mathcal{F}^{\mu \nu} \mathcal{F}^{\rho \sigma} \epsilon_{\mu \nu \rho \sigma}\right]_{S^{2}} .
$$

As shown in section 5.1, this can be absorbed in a renormalized action (5.9) for a suitable value of $\alpha \neq 1$.

Clearly the maximal supersymmetry of the model protects the flat limit $R \rightarrow \infty$ from large quantum corrections (i.e. from the non-local UV/R mixing), leading only to the above mild term. Note that there is no "cosmological constant" induced at one loop; in fact the very concept does not apply in this framework, which is based on matrix degrees of freedom rather than a fundamental metric. Only the background curvature (which we dropped) might lead to modifications in the linearized Einstein equation (5.37) which look like a cosmological constant. Hence the "cosmological constant" problem is replaced here by the question of stability of a background with sufficiently large $R$ and small extra dimensions. These are hopefully feasible problems which need to be addressed in future work.

\section{Conclusion and outlook}

We have shown that the 4-dimensional (Euclidean, linearized) Einstein equations emerge from the dynamics of fluctuations on fuzzy 4 -spheres $\mathcal{S}_{\Lambda}^{4}$ in Yang-Mills matrix models, in a certain regime and provided certain conditions are met. The resulting physics is richer than in general relativity, since there are several contributions to the metric. Most importantly, gravity is modified at long-distances, in the sense that wavelengths much larger than a certain scale $L_{R} / \alpha$ do not contribute to gravity. Moreover, a tower of higher-spin fields arises on top of the gravitational modes, leading to a higher-spin theory. The present analysis is expected to capture the leading gravitational effects, since fields with spin larger than 2 should decouple at low energies. Thus the gravitational physics of the present model could be sufficiently close to general relativity at least for solar-system scales.

The conditions to obtain an interesting gravity are as follows: 1) the background must be a generalized "thick" fuzzy sphere $\mathcal{S}_{\Lambda}^{4}$, leading to a large scale $L_{R} / \alpha$ which acts as an IR cutoff for gravity, and 2) dimensional reduction to 4 dimensions is justified. We discussed possible mechanisms to achieve this. One obvious mechanism involves the radial potential which stabilizes $\mathcal{S}_{N}^{4}$. Another possibility is to give VEV's to the transversal scalar fields along the lines of $[41,47]$, leading to fuzzy extra dimensions. This is natural given the structure of $\mathcal{S}_{\Lambda}^{4}$ as bundle over $\mathcal{S}_{N}^{4}$, and it would also provide an interesting symmetry breaking structure, leading to a low-energy gauge theory in the right ball-park of particle physics [42]. Yet another possibility is to have a self-dual Yang-Mills action ${ }^{18}$ (5.29); then $\alpha=0$, and the extra dimensions (i.e. $c_{n}$ ) may be small (but non-zero; the basic fuzzy

\footnotetext{
${ }^{18}$ There are indeed hints that this arises taking fully into account the volume fluctuations, cf. [7, 8].
} 
sphere $S_{N}^{4}$ does not suffice). Anyway, it is intriguing that the generalization to $\mathcal{S}_{\Lambda}^{4}$ seems to provides the required ingredients for both gravity and interesting particle physics.

To clarify these conditions requires a more detailed treatment of the generic fuzzy spheres $\mathcal{S}_{\Lambda}^{4}$ (cf. appendix A), as well as an understanding of the effective potential for the extra dimensions which would arise at one loop. Assuming that these conditions can be met, the long-wavelength modification of gravity discussed above could be very interesting, as they might lead to behavior usually attributed to dark matter or dark energy. There will also be new effects due to additional modes arising e.g. from radial deformations $\kappa$.

Apart from the above conditions, there are other issues which need to be addressed before physical implications can be extracted. One is to find a suitable Minkowski version of the background. While most of the analysis will generalize, the proper choice of a covariant Minkowskian matrix geometry is not clear, and there are non-trivial issues related to the non-compactness of the Lorentz group. ${ }^{19}$ Natural candidates would be based on a noncompact version of $\mathrm{SO}(6)$ (cf. [48]), or possibly some fuzzy de Sitter space [49, 50].

The restriction to linearized gravity in this paper is clearly not essential. The model is fully non-linear, and much of the derivation would go through for perturbations on a non-trivial background. We simply have to make the replacement (4.33) in the general mode expansion (3.16), and perturbations around a non-trivial $\gamma^{\mu \nu}$ could be studied along the same lines, leading presumably to the full Einstein equations on $\mathcal{S}_{\Lambda}^{4}$. Hence there is no obstacle for describing strong gravity in this manner.

For the IKKT matrix model, the quantization should be well-behaved, and the present mechanism provides a promising basis for a quantum theory of gravity with low-energy physics close to GR. The maximal supersymmetry protects backgrounds with large radius, and leads to a stabilization [36]. Moreover the non-local UV/IR mixing is mild in this model, and reduces to 10-dimensional supergravity in the bulk $[1,25,51]$. We have started this endeavour by computing the leading one-loop corrections for the simplest fuzzy 4sphere, which lead to modified parameters of the action including $\alpha$.

The relation of the IKKT model with IIB string theory also suggests an interesting general message: there is no need to compactify target space, so that the vast landscape of string compactifications may simply not be needed. While IIB supergravity arises in the bulk upon quantization, this has nothing to do with the present mechanism for gravity, which is purely classical. The present mechanism should therefore not be confused with mechanisms to localize bulk gravity to the brane such as [52]. If it is possible to obtain also a (near-) realistic low-energy particle physics in this framework (e.g. along the lines of $[41,42,47])$, it would provide an extremely simple and attractive approach to a quantum theory of fundamental interactions.

\section{Acknowledgments}

I would like to thank J. Barrett, S. Fredenhagen, M. Hanada, J. Karczmarek, H. Kawai for useful discussions, and S. Ramgoolam and J. Zahn for related discussions and collaboration.

\footnotetext{
${ }^{19}$ One problem is that the internal fiber could be noncompact as already noted in [18], hence the meaning of averaging is not clear. However, the expansion into higher spin modes would still go through.
} 
This work was supported by the Austrian Science Fund (FWF) grant P28590, and by the Action MP1405 QSPACE from the European Cooperation in Science and Techno$\operatorname{logy}(\operatorname{COST})$.

\section{A The classical geometry of the 4 -spheres $\mathcal{S}_{\Lambda}^{4}$}

The fuzzy 4-spheres under consideration are quantizations of the (co)adjoint orbits $\mathcal{O}[\Lambda]=$ $\left\{g \cdot H_{\Lambda} \cdot g^{-1} ; g \in \mathrm{SU}(4)\right\} \hookrightarrow \mathfrak{s u}(4)$ projected to $\mathbb{R}^{5}$ via the projection $\Pi$ (2.12),

$$
\mathcal{S}_{\Lambda}^{4}:=\Pi(\mathcal{O}[\Lambda]) \subset \mathbb{R}^{5} .
$$

The coadjoint orbit is a homogeneous space $\mathcal{O}[\Lambda] \cong \mathrm{SU}(4) / \mathcal{K}$ where $\mathcal{K}$ is the stabilizer of $\Lambda$. Here we discuss the classical geometry of these spaces and their harmonics. This is best understood in terms of the spinorial representation of $\mathfrak{s u}(4) \cong \mathfrak{s o}(6)$ on $\mathbb{C}^{4}$. Let $\gamma_{a}$ be $4 \times 4$ hermitian gamma matrices of $\mathrm{SO}(5)$ with $\left\{\gamma_{a}, \gamma_{b}\right\}=2 g_{a b}$ for $a, b=1, \ldots, 5$. To be specific, we choose the Weyl basis where

$$
\gamma_{5}=\left(\begin{array}{cc}
\mathbb{1}_{2} & 0 \\
0 & -\mathbb{1}_{2}
\end{array}\right) .
$$

Then a 4-dimensional representation of $\mathfrak{s o}(6)$ can be defined by the following generators

$$
\Sigma_{\mu \nu}:=\frac{1}{4 i}\left[\gamma_{\mu}, \gamma_{\nu}\right] \quad \Sigma_{\mu 5}:=-\frac{i}{2} \gamma_{\mu} \gamma_{5} \quad \Sigma_{\mu 6}:=-\frac{1}{2} \gamma_{\mu} \quad \Sigma_{56}:=-\frac{1}{2} \gamma_{5}
$$

where $\mu, \nu=1, \ldots, 4$. The embedding of $\mathcal{O}[\Lambda] \hookrightarrow \mathfrak{s o}(6)=\mathbb{R}^{15}$ is then described by the 15 (real-valued, commutative) embedding functions

$$
m^{a b}=\operatorname{tr}\left(\Xi \Sigma^{a b}\right), \quad a, b=1, \ldots, 6, \quad \Xi \in \mathcal{O}[\Lambda] .
$$

The point $\Xi \in \mathcal{O}[\Lambda]$ can then be recovered from

$$
\Xi=\sum_{1 \leq a<b \leq 6} m^{a b} \Sigma_{a b} \quad \in \mathcal{O}[\Lambda]
$$

In particular,

$$
x_{a}=\operatorname{tr}\left(\Xi \Sigma_{a 6}\right)=-\frac{1}{2} \operatorname{tr}\left(\Xi \gamma_{a}\right)
$$

(setting $r=1$ ) defines the embedding of $\mathcal{O}[\Lambda]$ in $\mathbb{R}^{5}$, which is the classical limit of $\mathcal{S}_{\Lambda}^{4}$.

The corresponding quantized ("fuzzy") coadjoint orbits are simply obtained by replacing the functions $m^{a b}$ on $\mathcal{O}[\Lambda]$ by the generators $\mathcal{M}^{a b}$ acting on the highest weight irrep $\mathcal{H}_{\Lambda}$, where $\Lambda$ should be a (dominant) integral weight. More details can be found e.g. in [30].

\section{A.1 The basic sphere $\mathcal{S}_{N}^{4}$}

The fuzzy sphere $\mathcal{S}_{N}^{4}$ is obtained for $\Lambda=N \Lambda_{1}=(0,0, N)$ or equivalently ${ }^{20}$

$$
H_{N} \equiv H_{N \Lambda_{1}}=N\left|\psi_{0}\right\rangle\left\langle\psi_{0}\right| \quad \text { for }\left|\psi_{0}\right\rangle=(1,0,0,0)^{T} \in \mathbb{C}^{4}
$$

\footnotetext{
${ }^{20}$ For better readability we do not impose tracelessness here. This does not lead to significant changes.
} 
The stabilizer of $\Lambda$ is $\mathcal{K}=\mathrm{SU}(3) \times \mathrm{U}(1)$, and clearly $\mathcal{O}[\Lambda] \cong \mathbb{C} P^{3}$. By inspection of (A.2) we find

$$
\begin{aligned}
& x_{\mu}=-\frac{1}{2} \operatorname{tr}\left(H_{\Lambda} \gamma_{\mu}\right)=0, \quad \mu=1, \ldots, 4 \\
& x_{5}=\frac{N}{2}=R_{N} .
\end{aligned}
$$

This defines our reference point $x_{(0)} \in S^{4}$ (the "north pole"). It is easy to see using $\mathrm{SO}(5)$ invariance and the explicit form of the generators (A.3) that

$$
\begin{aligned}
x_{a} x^{a} & \equiv \sum_{a=1}^{5} x_{a}^{2}=R_{N}^{2} \\
m_{a b} & =\frac{1}{2 R} \epsilon_{a b c d e} m^{c d} x^{e} \\
p_{\mu} & \propto m_{\mu 5}=\operatorname{tr}\left(H_{\Lambda} \Sigma_{\mu 5}\right)=0 .
\end{aligned}
$$

The second identity expresses self-duality. The stabilizer group of $x_{(0)}$ is

$$
\left\{h \in \mathrm{SO}(5) ;\left[h, \gamma_{5}\right]=0\right\}=\mathrm{SU}(2)_{R} \times \mathrm{SU}(2)_{L} \subset \mathrm{SO}(5)
$$

where $\mathrm{SU}(2)_{L}$ acts on the +1 eigenspace of $\gamma_{5}$. Hence there is a fiber of points $\mathbf{x} \in \mathbb{C} P^{3}$ over each point $x \in S^{4}$, which at the reference point $x_{(0)}$ is obtained by acting with $\mathrm{SU}(2)_{L}$ on $\left|\psi_{0}\right\rangle$. These $\mathrm{f}$ cibers are resolved by the functions $m_{\mu \nu}, \mu, \nu=1, \ldots, 4$, which define a tangential SD rank 2 tensor (or a 2 -form) on $S^{4}$ with

$$
m_{\mu \nu} m^{\mu \nu}=4 R_{N}^{2}
$$

These define 2 independent functions, which describe the internal $S^{2}$ fiber of $\mathcal{S}_{N}^{4} \cong \mathbb{C} P^{3}$ over $S^{4}$. However, the "momentum" functions $p_{\mu}$ vanish for any point on the fiber over $x$. Hence there are no independent modes $F_{\mu}(x) p^{\mu}$ on the basic sphere $\mathcal{S}_{N}^{4}$. Another way to see this is via the Poisson bracket identity

$$
0=\left\{x^{b} x_{b}, x^{a}\right\}=2 x_{b} m^{b a}
$$

since $x_{b} x^{b}=R_{N}^{2}$ for the basic fuzzy 4 -sphere (but not for the generalized ones). At the north pole, this gives $p^{\mu}=0$. Moreover, the following identity of $\mathfrak{s o}(6)$ tensors holds

$$
\sum_{a=1}^{6} m^{a b} m^{a c} \equiv \sum_{\mu=1}^{4} m^{\mu a} m^{\mu b}+x^{a} x^{b}=R_{N}^{2} \delta^{a b} .
$$

Besides direct verification, this follows (similar as in section B) from the fact that $\mathcal{C}^{\infty}\left(\mathbb{C} P^{3}\right)$ does not contain any $(0,2,0)$ modes, leaving only the trivial tensor $\delta^{b c}$ for the rhs. 


\section{A.2 The generalized sphere $\mathcal{S}_{\Lambda}^{4}$}

Now consider $\mathcal{S}_{\Lambda}^{4}$ for $\Lambda=N \Lambda_{1}+n_{1} \Lambda_{1}^{\prime}+n_{2} \Lambda_{2}^{\prime}$, where $\Lambda_{i}^{\prime}$ are fundamental weights of the $\mathfrak{s u}(3)$ stabilizator of $\Lambda_{1}$ (hence orthogonal to $\Lambda_{1}$ ), for $n_{1}, n_{2} \ll N$. Then

$$
\begin{aligned}
H_{\Lambda}=H_{N \Lambda_{1}}+ & H_{n_{1}, n_{2}}^{\prime}=N\left|\psi_{0}\right\rangle\left\langle\psi_{0}\left|+\sum_{i=1,2} n_{i}\right| \psi_{i}\right\rangle\left\langle\psi_{i}\right|, \\
\left\langle\psi_{i} \mid \psi_{j}\right\rangle & =\delta_{i j} .
\end{aligned}
$$

Clearly $H_{\Lambda}$ satisfies a characteristic equation

$$
\chi\left(H_{\Lambda}\right)=\left(H_{\Lambda}-N\right) P\left(H_{\Lambda}\right)=0
$$

with one large eigenvalue $N$, and one or two eigenvalues $n_{i}$ encoded in the polynomial $P\left(H_{\Lambda}\right)$. Since $P$ maps $n_{i}$ to 0 , it follows that $P$ (extended to the entire SU(4) orbit) is essentially a projector, which projects $\mathcal{O}[\Lambda]$ to $\mathcal{O}\left[N \Lambda_{1}\right] \cong \mathbb{C} P^{3}$. Geometrically, this means that the generic orbits $\mathcal{O}[\Lambda]$ are naturally bundles over $\mathbb{C} P^{3}$,

$$
\begin{aligned}
& \mathcal{O}[\Lambda] \\
& P \downarrow \\
& \mathcal{O}\left[N \Lambda_{1}\right] \cong \mathbb{C} P^{3} \stackrel{x^{a}}{\rightarrow} S^{4} \hookrightarrow \mathbb{R}^{5} .
\end{aligned}
$$

The fibers of this bundle are given by the $\mathfrak{s u}(3)$ coadjoint orbits $\mathcal{O}_{n}:=\left\{U H_{n_{i}}^{\prime} U^{-1}, U \in\right.$ $\mathrm{SU}(3)\}$, which are resolved by the functions $p^{\mu}$ and $m^{\mu \nu}$ on $\mathcal{O}[\Lambda]$. More precisely, for $n_{2}=0$ this is the 4-dimensional space $\mathcal{O}_{n} \cong \mathbb{C} P^{2}$ parametrized by $p^{\mu}$, while $m^{\mu \nu}$ is still self-dual and describes the $S^{2}$ fiber of $\mathbb{C} P^{3}$ over $S^{4}$. For $n_{1}, n_{2} \neq 0, \mathcal{O}_{n}$ is a 6 -dimensional coadjoint orbit of $\mathfrak{s u}(3)$ parametrized by $p^{\mu}$ and the ASD components of $m^{\mu \nu}$. For simplicity we assume $n_{2}=0$, and $H_{\Lambda}=H_{N \Lambda_{1}}+H_{n}^{\prime}$ where $H_{n}^{\prime}=U \operatorname{diag}(0, n, 0,0) U^{-1}$ for $U \in \mathrm{SU}(3)$. Then

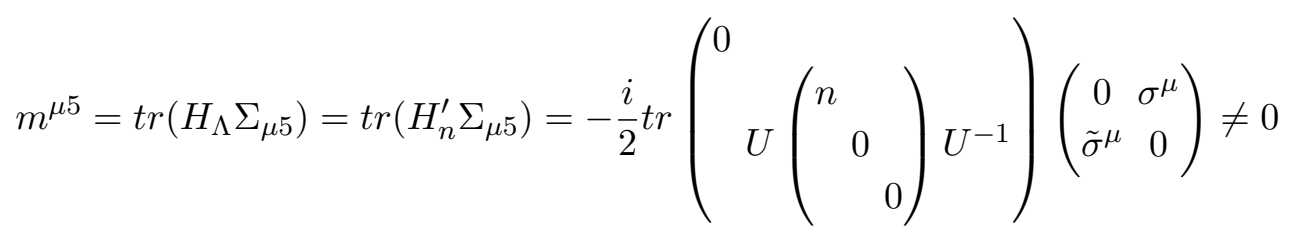

which is not constant along $\mathcal{O}_{n}$. Upon averaging over the local fiber, one obtains

$$
\left[m_{\mu 5} m^{\mu 5}\right]_{0}=c_{n}^{2}=O\left(n^{2}\right)>0
$$

(which we refrain from computing here explicitly). Hence in contrast to the basic $\mathcal{S}_{N}^{4}$, the "momentum" functions $p^{\mu}$ are independent, so that the modes $F_{\mu}(x) p^{\mu}$ are non-trivial. Similarly, the radial function

$$
\begin{aligned}
\mathcal{R}^{2}=x_{a} x^{a} & =\frac{1}{4} \operatorname{tr}\left(\Xi \otimes \Xi \gamma_{a} \otimes \gamma^{a}\right)=\frac{1}{4} \operatorname{tr}\left(\Xi \otimes \Xi\left(-\mathbb{1}+2 P+8 P_{1}\right)\right) \\
& =\frac{1}{4}\left(-(\operatorname{tr} \Xi)^{2}+2 \operatorname{tr}\left(\Xi^{2}\right)+16 N n\left(\epsilon \bar{\psi}_{0} \bar{\psi}_{1}\right)\left(\epsilon \psi_{0} \psi_{1}\right)\right) \\
& =\frac{1}{4}\left(-(N+n)^{2}+2\left(N^{2}+n^{2}\right)+16 N n|\tau|^{2}\right), \quad \tau=\epsilon \psi_{0} \psi_{1} \quad \in \mathbb{C} \\
& =\frac{1}{4}\left(N^{2}-N n+n^{2}+16 N n|\tau|^{2}\right), \quad \tau=\epsilon \psi_{0} \psi_{1} \quad \in \mathbb{C}
\end{aligned}
$$


for $\Xi \in \mathcal{O}[\Lambda]$ using (B.1), where $P$ is the permutation operator acting on $\mathbb{C}^{4} \otimes \mathbb{C}^{4}$. Now the point is that $\tau$ is not invariant under $\mathrm{SO}(6)$, so that the spectrum of $\mathcal{R}^{2}$ lies in an interval $\left[R_{\min }^{2}, R_{\max }^{2}\right]$ peaked around $R_{N}^{2}=\frac{N^{2}}{4}$. This means that the generic 4-spheres $\mathcal{S}_{\Lambda}^{4}$ are "thick" spheres, with $\left\{\mathcal{R}^{2}, x^{a}\right\} \neq 0$. This essential for the existence of independent momentum functions $p^{\mu}$, which are the basis of the present mechanism for gravity. Finally we note that the identity (A.13) still holds approximately, in the form

$$
\sum_{\mu=1}^{4} m^{\mu a} m^{\mu b}=R_{N}^{2}\left(P_{T}^{a b}+t^{a b}\right)
$$

where $P_{T}^{a b}=\delta^{a b}-\frac{1}{R_{N}^{2}} x^{a} x^{b}$ is the tangential projector on $S^{4} \subset \mathbb{R}^{5}$, and $t^{a b}=O\left(\frac{n}{N}\right)$ arises from $(0,2,0)$ modes in $\mathcal{C}^{\infty}\left(\mathcal{S}_{\Lambda}^{4}\right)$.

\section{B Some identities for fuzzy 4-spheres}

First, we note the following identity for the $\mathrm{SO}(5)$ gamma matrices

$$
\gamma_{a} \otimes \gamma^{a}=\frac{1}{2}(\mathbb{1}+P)-\frac{3}{2}(\mathbb{1}-P)+8 P_{1}
$$

Here $P_{1}=\bar{\epsilon} \epsilon$ is the projector on the $\mathfrak{s o}(5)$ singlet in $(4) \otimes(4)=\left((10)_{S} \oplus(6)_{A S}\right)_{\mathfrak{s o}(6)}=$ $\left((10)_{S} \oplus(5)_{A S} \oplus(1)_{A S}\right)_{\mathfrak{s o}(5)}$, which is broken by $\mathfrak{s o}(6)$. Furthermore, we are interested in the following tensor operator

$$
\mathcal{T}^{a b}:=\frac{1}{2} \sum_{a, a^{\prime}=1}^{6}\left\{\mathcal{M}^{a b}, \mathcal{M}^{a^{\prime} c}\right\}_{+} \delta_{a a^{\prime}} .
$$

Consider first

The basic fuzzy sphere $\mathcal{S}_{N}^{4}$. Since $\operatorname{End}(\mathcal{H})$ does not contain any $(0,2,0)$ modes, it follows ${ }^{21}$ that $\mathcal{T}^{a b} \sim \delta^{a b}$. Computing the trace $\mathcal{T}=\mathcal{T}^{a b} \delta_{a b}=2 C^{2}[\mathfrak{s o}(6)]=\frac{3}{2} N(N+4)$ (cf. [36]), we obtain

$$
\mathcal{T}^{a b}=\frac{1}{3} C^{2}[\mathfrak{s o}(6)] \delta^{b c}=R_{N}^{2} \delta^{b c}
$$

i.e.

$$
\frac{1}{2} \sum_{a, a^{\prime}=1}^{5}\left\{\mathcal{M}^{a b}, \mathcal{M}^{a^{\prime} c}\right\} g_{a a^{\prime}}=R_{N}^{2} g^{b c}-\frac{1}{2}\left\{X^{b}, X^{c}\right\} .
$$

This is the fuzzy analog of (A.13). For the

Generalized fuzzy spheres $\mathcal{S}_{\Lambda}^{4}$ with $\Lambda=\left(n_{1}, n_{2}, N\right)$, End $(\mathcal{H})$ may contain some $(0,2,0)$ modes. Then the above relation generalizes as

$$
\frac{1}{2} \sum_{a, a^{\prime}=1}^{6}\left\{\mathcal{M}^{a b}, \mathcal{M}^{a^{\prime} c}\right\} \delta_{a a^{\prime}}=\frac{1}{3} \delta^{b c} C^{2}[\mathfrak{s o}(6)]+t^{a b}
$$

where $t^{a b}$ is a traceless $(0,2,0)$ tensor operator of order $t^{a b}=O(n) \ll C^{2}[\mathfrak{s o}(6)]$, which is suppressed. This is the fuzzy analog of (A.20).

\footnotetext{
${ }^{21}$ Note that $(0,1,0)$ is the 6 -dimensional vector representation of $\mathfrak{s o}(6)$.
} 


\section{Background flux $\theta^{\mu \nu}(x, \xi)$ averaged over $S^{2}$}

We need various averages of the background flux $\theta^{\mu \nu}(x, \xi)$ over $S^{2}$. One useful result which follows from the self-duality and (2.35) is

$$
\left[\theta^{\mu \nu} \theta^{\rho \sigma}\right]_{0}=\frac{\Delta^{4}}{12}\left(\delta^{\mu \rho} \delta^{\nu \sigma}-\delta^{\nu \rho} \delta^{\mu \sigma}+\varepsilon^{\mu \nu \rho \sigma}\right)
$$

This also applies to $\mathcal{S}_{N}^{4}$, and to $\mathcal{S}_{\Lambda}^{4}$ as long as $N \gg n_{i}$. Furthermore since $\theta^{\mu \nu}$ is self-dual, we can write

$$
\theta^{\mu \nu}(\xi)=r^{2} \theta_{a}^{\mu \nu} J^{a}(\xi)
$$

where $J^{a}$ are the generators of the internal fuzzy sphere $S_{N+1}^{2}$, which in the semi-classical limit are functions $J^{a}: S^{2} \rightarrow \mathbb{R}^{3}$ on $S^{2}$ with radius given by

$$
\theta^{\mu \nu} \theta_{\mu \nu}=4 r^{4} J^{a} J_{a} \sim N^{2} r^{4}
$$

using

$$
\theta_{a}^{\mu \nu} \theta_{\mu \nu}^{b}=4 \delta_{a}^{b}
$$

Therefore

$$
\begin{aligned}
m^{2}(\xi, \eta) & =\left(\theta^{\mu \nu}(\xi)-\theta^{\mu \nu}(\eta)\right)\left(\theta_{\mu \nu}(\xi)-\theta_{\mu \nu}(\eta)\right) \\
& =4 r^{4}\left(J^{a}(\xi)-J^{a}(\eta)\right)\left(J_{a}(\xi)-J_{a}(\eta)\right) \\
& \sim \Delta^{4}\|\xi-\eta\|^{2}
\end{aligned}
$$

where $\xi, \eta$ are unit vectors on $S^{2}$, and recalling $N^{2} r^{4}=\Delta^{4}$.

\section{Mixed Young projections and permutations}

Define

$$
\begin{aligned}
P_{\text {hor }}: & =\frac{1-P_{23}}{2} P_{12} \frac{1-P_{23}}{2} \\
P_{\text {hor }}^{2} & =-\frac{1}{2} P_{\text {hor }}+\left(1-P_{23}\right) .
\end{aligned}
$$

Acting on tensors which are anti-symmetric in the last two indices we have $P_{23}=-1$, and

$$
\left(P_{\text {hor }}+1\right)\left(P_{\text {hor }}-\frac{1}{2}\right)=0 .
$$

Hence solutions of $P_{\text {hor }}=-1$ are the totally anti-symmetric Young diagrams, while the solutions of $P_{\text {hor }}=\frac{1}{2}$ are mixed (hook) Young diagrams $A_{\mu \rho \sigma}$. This means that interchanging the first two ("horizontal") indices of such $A_{\mu \rho \sigma}$ costs a factor $\frac{1}{2}$. 


\section{E Evaluation of $D^{2}$}

First, one easily derives from the basic $\mathcal{S}_{N}^{4}$ algebra the following semi-classical results

$$
\begin{aligned}
\square \theta^{\mu \nu}= & 2 r^{2} \theta^{\mu \nu}, \quad \square P^{\mu}=2 r^{2} P^{\mu} \\
\square\left(\theta^{\mu \nu} P^{\sigma}\right)= & \square \theta^{\mu \nu} P^{\sigma}+\theta^{\mu \nu} \square P^{\sigma}+2\left[X^{\alpha}, \theta^{\mu \nu}\right]\left[X_{\alpha}, P^{\sigma}\right]=4 r^{2} \theta^{\mu \nu} P^{\sigma} \\
\square\left(\theta^{\mu \nu} \mathcal{M}^{\sigma \rho}\right)= & 4 r^{2} \theta^{\mu \nu} \mathcal{M}^{\sigma \rho} \\
2 i\left[\theta^{\mu \mu^{\prime}}, \theta^{\mu^{\prime} \nu} P^{\sigma}\right]= & 4 r^{2} \theta^{\mu \nu} P^{\sigma}+2 \mathcal{M}^{\sigma \nu} P^{\mu} \\
2 i\left[\theta^{\mu \mu^{\prime}}, \theta^{\mu^{\prime} \nu} \mathcal{M}^{\sigma \rho}\right]= & -2 r^{2}\left(g^{\mu \mu^{\prime}} \theta^{\mu^{\prime} \nu}-g^{\mu^{\prime} \mu^{\prime}} \theta^{\mu \nu}+g^{\mu^{\prime} \nu} \theta^{\mu \mu^{\prime}}\right) \mathcal{M}^{\sigma \rho} \\
& \quad-2 \theta^{\mu^{\prime} \nu}\left(g^{\mu \sigma} \theta^{\mu^{\prime} \rho}-g^{\mu \rho} \theta^{\mu^{\prime} \sigma}-g^{\mu^{\prime} \sigma} \theta^{\mu \rho}+g^{\mu^{\prime} \rho} \theta^{\mu \sigma}\right) \\
= & 4 r^{2} \theta^{\mu \nu} \mathcal{M}^{\sigma \rho}-2\left(\gamma^{\nu \rho} g^{\mu \sigma}-\gamma^{\nu \sigma} g^{\mu \rho}-\theta^{\mu \rho} \theta^{\sigma \nu}+\theta^{\mu \sigma} \theta^{\rho \nu}\right) \\
= & 2\left(2 \theta^{\mu \nu} \theta^{\sigma \rho}+\theta^{\mu \rho} \theta^{\sigma \nu}-\theta^{\mu \sigma} \theta^{\rho \nu}\right)+2\left(g^{\mu \rho} \gamma^{\nu \sigma}-g^{\mu \sigma} \gamma^{\nu \rho}\right)
\end{aligned}
$$

noting that $\theta^{\mu \nu} P_{\mu}=0$ at $p$. As a check, consider ${ }^{22}$

$$
\begin{aligned}
2 i\left[\theta^{\mu \mu^{\prime}}, \theta^{\mu^{\prime} \nu} g_{\nu \sigma} \mathcal{M}^{\sigma \rho}\right] & =2\left(2 \theta^{\mu \nu} \theta^{\sigma \rho}-\theta^{\mu \sigma} \theta^{\rho \nu}\right)+2\left(g^{\mu \rho} \gamma^{\nu \sigma}-g^{\mu \sigma} \gamma^{\nu \rho}\right) g_{\nu \sigma} \\
& =2\left(-2 \gamma^{\mu \rho}-\gamma^{\mu \rho}\right)+6 \gamma^{\mu \rho}=0 .
\end{aligned}
$$

Using these results and the semi-classical rules (5.3) we obtain

$$
\begin{aligned}
\square\left(\theta^{\mu \nu} A_{\nu \sigma}(x) P^{\sigma}\right) & =\left[X^{a},\left[X_{a}, \theta^{\mu \nu} A_{\nu \sigma} P^{\sigma}\right]\right] \\
& \sim\left(\square+4 r^{2}\right) A_{\nu \sigma} \theta^{\mu \nu} P^{\sigma}+2 \theta^{\mu \nu} \theta^{\alpha \sigma} \partial_{\alpha} A_{\nu \sigma} \\
\square\left(\theta^{\mu \nu} A_{\nu \sigma \rho}(x) \mathcal{M}^{\sigma \rho}\right) & =\square A_{\nu \sigma \rho} \theta^{\mu \nu} \mathcal{M}^{\sigma \rho}+A_{\nu \sigma \rho} \square\left(\theta^{\mu \nu} \mathcal{M}^{\sigma \rho}\right)+2\left[X^{a}, \theta^{\mu \nu} \mathcal{M}^{\sigma \rho}\right]\left[X_{a}, A_{\nu \sigma \rho}\right] \\
& \sim\left(\square+4 r^{2}\right) A_{\nu \sigma \rho} \theta^{\mu \nu} \mathcal{M}^{\sigma \rho}
\end{aligned}
$$

always dropping terms like $\left[X^{\alpha}, \theta^{\mu \nu}\right] \sim x=0$ at $p$, so that e.g. $\left[X^{\alpha}, \theta^{\mu \nu} P^{\sigma}\right] \sim-i \theta^{\mu \nu} g^{\alpha \sigma}$.

Open Access. This article is distributed under the terms of the Creative Commons Attribution License (CC-BY 4.0), which permits any use, distribution and reproduction in any medium, provided the original author(s) and source are credited.

\section{References}

[1] N. Ishibashi, H. Kawai, Y. Kitazawa and A. Tsuchiya, A large-N reduced model as superstring, Nucl. Phys. B 498 (1997) 467 [hep-th/9612115] [INSPIRE].

[2] T. Banks, W. Fischler, S.H. Shenker and L. Susskind, $M$ theory as a matrix model: A conjecture, Phys. Rev. D 55 (1997) 5112 [hep-th/9610043] [INSPIRE].

[3] B. de Wit, J. Hoppe and H. Nicolai, On the Quantum Mechanics of Supermembranes, Nucl. Phys. B 305 (1988) 545 [INSPIRE].

[4] S.-W. Kim, J. Nishimura and A. Tsuchiya, Expanding (3+1)-dimensional universe from a Lorentzian matrix model for superstring theory in (9+1)-dimensions, Phys. Rev. Lett. 108 (2012) 011601 [arXiv:1108.1540] [INSPIRE].

\footnotetext{
${ }^{22}$ Note that $\square\left(\theta^{\mu \nu} \mathcal{M}^{\sigma \rho}\right)$ is not consistent with a contraction by $g_{\nu \sigma}$, i.e. its trivial component $\left[\theta^{\mu \nu} \mathcal{M}^{\sigma \rho}\right]_{S^{2}}$ would require to keep sub-leading terms. However this correction is not significant.
} 
[5] S.-W. Kim, J. Nishimura and A. Tsuchiya, Late time behaviors of the expanding universe in the IIB matrix model, JHEP 10 (2012) 147 [arXiv:1208.0711] [INSPIRE].

[6] A. Connes, M.R. Douglas and A.S. Schwarz, Noncommutative geometry and matrix theory: Compactification on tori, JHEP 02 (1998) 003 [hep-th/9711162] [INSPIRE].

[7] H. Steinacker, Emergent Gravity from Noncommutative Gauge Theory, JHEP 12 (2007) 049 [arXiv: 0708.2426] [INSPIRE].

[8] H. Steinacker, Emergent Geometry and Gravity from Matrix Models: an Introduction, Class. Quant. Grav. 27 (2010) 133001 [arXiv:1003.4134] [INSPIRE].

[9] H.S. Yang, Emergent Gravity from Noncommutative Spacetime, Int. J. Mod. Phys. A 24 (2009) 4473 [hep-th/0611174] [INSPIRE].

[10] H.S. Yang and M. Sivakumar, Emergent Gravity from Quantized Spacetime, Phys. Rev. D 82 (2010) 045004 [arXiv: 0908. 2809] [INSPIRE].

[11] V.O. Rivelles, Noncommutative field theories and gravity, Phys. Lett. B 558 (2003) 191 [hep-th/0212262] [INSPIRE].

[12] R.J. Szabo, Symmetry, gravity and noncommutativity, Class. Quant. Grav. 23 (2006) R199 [hep-th/0606233] [INSPIRE].

[13] R.J. Szabo, Quantum Gravity, Field Theory and Signatures of Noncommutative Spacetime, Gen. Rel. Grav. 42 (2010) 1 [arXiv:0906.2913] [InSPIRE].

[14] P.-M. Ho and S. Ramgoolam, Higher dimensional geometries from matrix brane constructions, Nucl. Phys. B 627 (2002) 266 [hep-th/0111278] [INSPIRE].

[15] H.S. Snyder, Quantized space-time, Phys. Rev. 71 (1947) 38 [InSPIRE].

[16] M.V. Battisti and S. Meljanac, Scalar Field Theory on Non-commutative Snyder Space-Time, Phys. Rev. D 82 (2010) 024028 [arXiv: 1003.2108] [InSPIRE].

[17] F. Girelli and E.R. Livine, Scalar field theory in Snyder space-time: Alternatives, JHEP 03 (2011) 132 [arXiv: 1004.0621] [InSPIRE].

[18] S. Doplicher, K. Fredenhagen and J.E. Roberts, The quantum structure of space-time at the Planck scale and quantum fields, Commun. Math. Phys. 172 (1995) 187 [hep-th/0303037] [INSPIRE].

[19] M. Hanada, H. Kawai and Y. Kimura, Describing curved spaces by matrices, Prog. Theor. Phys. 114 (2006) 1295 [hep-th/0508211] [INSPIRE].

[20] S.W. MacDowell and F. Mansouri, Unified Geometric Theory of Gravity and Supergravity, Phys. Rev. Lett. 38 (1977) 739 [Erratum ibid. 38 (1977) 1376] [INSPIRE].

[21] D.K. Wise, MacDowell-Mansouri gravity and Cartan geometry, Class. Quant. Grav. 27 (2010) 155010 [gr-qc/0611154] [INSPIRE].

[22] M. Chaichian, A. Tureanu and G. Zet, Corrections to Schwarzschild solution in noncommutative gauge theory of gravity, Phys. Lett. B 660 (2008) 573 [arXiv:0710.2075] [INSPIRE].

[23] A.H. Chamseddine, Deforming Einstein's gravity, Phys. Lett. B 504 (2001) 33 [hep-th/0009153] [INSPIRE].

[24] M.A. Cardella and D. Zanon, Noncommutative deformation of four-dimensional Einstein gravity, Class. Quant. Grav. 20 (2003) L95 [hep-th/0212071] [INSPIRE]. 
[25] H.C. Steinacker, String states, loops and effective actions in noncommutative field theory and matrix models, Nucl. Phys. B 910 (2016) 346 [arXiv:1606.00646] [InSPIRE].

[26] C.N. Yang, On quantized space-time, Phys. Rev. 72 (1947) 874 [InSPIRE].

[27] H. Grosse, C. Klimčík and P. Prešnajder, On finite 4-D quantum field theory in noncommutative geometry, Commun. Math. Phys. 180 (1996) 429 [hep-th/9602115] [INSPIRE].

[28] J. Castelino, S. Lee and W. Taylor, Longitudinal five-branes as four spheres in matrix theory, Nucl. Phys. B 526 (1998) 334 [hep-th/9712105] [InSPIRE].

[29] S. Ramgoolam, On spherical harmonics for fuzzy spheres in diverse dimensions, Nucl. Phys. B 610 (2001) 461 [hep-th/0105006] [INSPIRE].

[30] E. Hawkins, Quantization of equivariant vector bundles, Commun. Math. Phys. 202 (1999) 517 [q-alg/9708030] [INSPIRE].

[31] J. Medina and D. O'Connor, Scalar field theory on fuzzy $S^{4}$, JHEP 11 (2003) 051 [hep-th/0212170] [INSPIRE].

[32] M. Fabinger, Higher dimensional quantum Hall effect in string theory, JHEP 05 (2002) 037 [hep-th/0201016] [INSPIRE].

[33] Y. Abe, Construction of fuzzy $S^{4}$, Phys. Rev. D 70 (2004) 126004 [hep-th/0406135] [INSPIRE].

[34] D. Karabali and V.P. Nair, Quantum Hall effect in higher dimensions, matrix models and fuzzy geometry, J. Phys. A 39 (2006) 12735 [hep-th/0606161] [INSPIRE].

[35] J. Medina, I. Huet, D. O'Connor and B.P. Dolan, Scalar and Spinor Field Actions on Fuzzy $S^{4}$ : fuzzy $C P^{3}$ as a $S_{F}^{2}$ bundle over $S_{F}^{4}$, JHEP 08 (2012) 070 [arXiv:1208.0348] [InSPIRE].

[36] H.C. Steinacker, One-loop stabilization of the fuzzy four-sphere via softly broken SUSY, JHEP 12 (2015) 115 [arXiv:1510.05779] [INSPIRE].

[37] D.N. Blaschke and H. Steinacker, On the 1-loop effective action for the IKKT model and non-commutative branes, JHEP 10 (2011) 120 [arXiv:1109.3097] [INSPIRE].

[38] H. Steinacker, Gravity and compactified branes in matrix models, JHEP 07 (2012) 156 [arXiv: 1202.6306] [INSPIRE].

[39] H. Steinacker, The curvature of branes, currents and gravity in matrix models, JHEP 01 (2013) 112 [arXiv:1210.8364] [INSPIRE].

[40] P. Aschieri, T. Grammatikopoulos, H. Steinacker and G. Zoupanos, Dynamical generation of fuzzy extra dimensions, dimensional reduction and symmetry breaking, JHEP 09 (2006) 026 [hep-th/0606021] [INSPIRE].

[41] H.C. Steinacker and J. Zahn, Self-intersecting fuzzy extra dimensions from squashed coadjoint orbits in $\mathcal{N}=4$ SYM and matrix models, JHEP 02 (2015) 027 [arXiv:1409.1440] [INSPIRE].

[42] H.C. Steinacker, Chiral low-energy physics from squashed branes in deformed $\mathcal{N}=4 S Y M$, JHEP 10 (2015) 119 [arXiv: 1504.05703] [INSPIRE].

[43] H. Grosse and H. Steinacker, Finite gauge theory on fuzzy $C P^{2}$, Nucl. Phys. B 707 (2005) 145 [hep-th/0407089] [INSPIRE].

[44] R.M. Wald, General Relativity, University of Chicago Press, U.S.A. (1984). 
[45] I. Chepelev and A.A. Tseytlin, Interactions of type IIB D-branes from D instanton matrix model, Nucl. Phys. B 511 (1998) 629 [hep-th/9705120] [INSPIRE].

[46] A.A. Tseytlin, Born-Infeld action, supersymmetry and string theory, hep-th/9908105 [INSPIRE].

[47] H.C. Steinacker and J. Zahn, An extended standard model and its Higgs geometry from the matrix model, PTEP 2014 (2014) 083B03 [arXiv: 1401.2020] [INSPIRE].

[48] J. Heckman and H. Verlinde, Covariant non-commutative space-time, Nucl. Phys. B 894 (2015) 58 [arXiv: 1401.1810] [INSPIRE].

[49] J.-P. Gazeau and F. Toppan, A natural fuzzyness of de Sitter space-time, Class. Quant. Grav. 27 (2010) 025004 [arXiv: 0907.0021 ] [INSPIRE].

[50] M. Burić and J. Madore, Noncommutative de Sitter and FRW spaces, Eur. Phys. J. C 75 (2015) 502 [arXiv : 1508.06058] [INSPIRE].

[51] M.R. Douglas and W. Taylor, Branes in the bulk of Anti-de Sitter space, hep-th/9807225 [INSPIRE].

[52] L. Randall and R. Sundrum, An alternative to compactification, Phys. Rev. Lett. 83 (1999) $4690[$ hep-th/9906064] [INSPIRE]. 